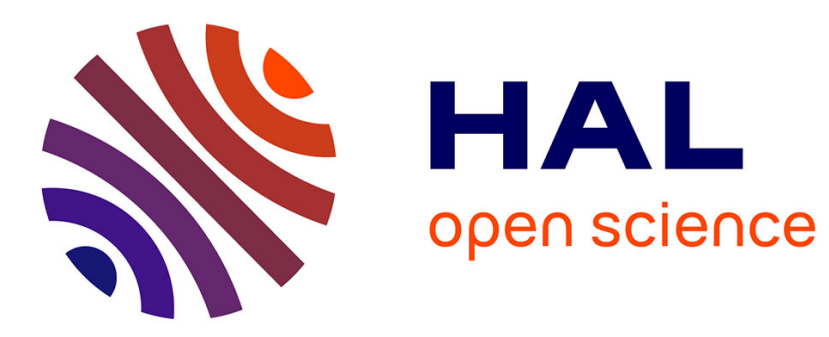

\title{
Local triviality for G-torsors
}

Philippe Gille, Raman Parimala, V. Suresh

\section{To cite this version:}

| Philippe Gille, Raman Parimala, V. Suresh. Local triviality for G-torsors. 2019. hal-01978093v4

\section{HAL Id: hal-01978093 \\ https://hal.science/hal-01978093v4}

Preprint submitted on 18 Mar 2020 (v4), last revised 1 Feb 2021 (v6)

HAL is a multi-disciplinary open access archive for the deposit and dissemination of scientific research documents, whether they are published or not. The documents may come from teaching and research institutions in France or abroad, or from public or private research centers.
L'archive ouverte pluridisciplinaire HAL, est destinée au dépôt et à la diffusion de documents scientifiques de niveau recherche, publiés ou non, émanant des établissements d'enseignement et de recherche français ou étrangers, des laboratoires publics ou privés. 


\title{
LOCAL TRIVIALITY FOR $G$-TORSORS
}

\author{
P. GILLE, R. PARIMALA, AND V. SURESH
}

\begin{abstract}
Let $C \rightarrow \operatorname{Spec}(R)$ be a relative proper flat curve over an henselian base. Let $G$ be a reductive $C$-group scheme. Under mild technical assumptions, we show that a $G$-torsor over $C$ which is trivial on the closed fiber of $C$ is locally trivial for the Zariski topology.

Keywords: Reductive group scheme, torsor, deformation.
\end{abstract}

MSC 2000: 14D23, 14F20.

\section{INTRODUCTION}

The purpose of the paper is to study local triviality for $G$-torsors over a proper flat relative curve $C$ over an affine base $S=\operatorname{Spec}(R)$. We deal here with semisimple $C$-group schemes which are not necessarily extended from $R$. Our main result can be stated as follows.

Theorem 1.1. Assume that $R$ is local henselian noetherian of residue field $\kappa$. Let $f: C \rightarrow \operatorname{Spec}(R)$ be a flat projective curve such that $C$ is integral. We assume that one of the following holds:

(I) $C$ is smooth with geometrically connected fibers;

(II) $R$ is a DVR and the map $\mathcal{O}_{S} \rightarrow f_{*} \mathcal{O}_{C}$ is universally an isomorphism.

Let $G$ be a semisimple $C$-group scheme and denote by $q: G^{s c} \rightarrow G$ its simply connected covering. We assume that the fundamental group $\mu / C=\operatorname{ker}(q)$ of $G$ is étale. Then the following hold:

(1) Let $E$ be a $G$-torsor over $C$ such that $E \times{ }_{C} C_{\kappa}$ is trivial. Then $E$ is locally trivial for the Zariski topology.

(2) Let $E, E^{\prime}$ be two $G$-torsors over $C$ such that $E \times{ }_{C} C_{\kappa}$ is isomorphic to $E^{\prime} \times_{C} C_{\kappa}$. Then $E$ and $E^{\prime}$ are locally isomorphic for the Zariski topology.

Remark 1.2. Note that $\mathcal{O}_{S} \rightarrow f_{*} \mathcal{O}_{C}$ is universally an isomorphism in case (I) and that case (II) includes the case when $C$ is normal, $\mathcal{O}_{S} \rightarrow f_{*} \mathcal{O}_{X}$ is an isomorphism and the g.c.d.of the geometric multiplicities of the irreducible components of $C_{\kappa}$ is prime to the characteristic exponent of $k$.

Date: March 18, 2020. 
A related result is that of Drinfeld and Simpson [D-S, th. 2]. In the case $G$ is semisimple split simply connected and $R$ is strictly henselian they showed in case (I) that a $G$-torsor over $C$ is locally trivial for the Zariski topology. Drinfeld-Simpson's result has been generalized recently by Belkale-Fakhruddin to a wider setting [B-F1, B-F2. We provide a variant in Theorem 6.4 in the case of a henselian base.

One important difference is that we only require that the ring $R$ is henselian. We consider Zariski triviality on $C$ with respect to henselian (or Nisnevich) topology on the base while Drinfeld-Simpson deal with Zariski triviality on $C$ with respect to the étale topology on the base. We stated the semisimple case but the result extends in the reductive case by combining with the case of tori, see Theorem 7.1 . We denote by $F$ the function field of $C$; in the case of a DVR, the main result leads to new cases of a local-global principle for $G_{F}$-torsors (Corollary 6.8). More precisely if $C$ is smooth over $R$ with geometrically connected fibers and $G$ a semisimple simply connected group over $C$, then a torsor under $G$ over $F$ which is trivial at all completions of $F$ at discrete valuations of $F$ is trivial.

Let us review the contents of the paper. The toral case is quite different from the semisimple one since it works in higher dimensions; it is treated in section 2 by means of the proper base change theorem. The section 3 deals with generation by one parameter subgroups, namely the Kneser-Tits problem. Section 4 extends Sorger's construction of the moduli stack of $G$-bundles $[\mathrm{So}]$ and discusses in details its tangent bundle. The next section 5 recollects facts on patching for $G$-torsors and provides the main technical statement namely the parametrization of the deformations of a given torsor in the henselian case in presence of isotropy (Proposition 5.4); this refines Heinloth's uniformization [He1]. Section 6] explain why this intermediate statement is enough for establishing that deformations of a given torsor (in the henselian case) are locally trivial for the Zariski topology. One important point is that we can get rid of isotropy assumptions. Finally section 7 provides a general theorem for reductive groups. We include at the end a short appendix 8 gathering facts on smoothness for morphisms of algebraic stacks.

Acknowledgements. We thank Laurent Moret-Bailly for the extension of the toral case beyond curves, a strengthened version of Lemma 6.2 and several suggestions. We thank Jean-Louis Colliot-Thélène for communicating to us his method to deal with tori. We thank Olivier Benoist for raising a question answered by Theorem 6.4.

Finally we thank Lie Fu, Ofer Gabber, Jochen Heinloth and Anastasia Stavrova for useful discussions.

The first author is supported by the project ANR Geolie, ANR-15-CE 40-0012, (The French National Research Agency). The second and third authors are partially supported by National Science Foundation grants DMS-1463882 and DMS-1801951.

Conventions and Notations. We use mainly the terminology and notations of Grothendieck-Dieudonné [EGAI, §9.4 and 9.6] which agrees with that of DemazureGrothendieck used in [SGA3, Exp. I.4] 
(a) Let $S$ be a scheme and let $\mathcal{E}$ be a quasi-coherent sheaf over $S$. For each morphism $f: T \rightarrow S$, we denote by $\mathcal{E}_{(T)}=f^{*}(\mathcal{E})$ the inverse image of $\mathcal{E}$ by the morphism $f$. We denote by $\mathbf{V}(\mathcal{E})$ the affine $S$-scheme defined by $\mathbf{V}(\mathcal{E})=\operatorname{Spec}(\operatorname{Sym} \bullet \mathcal{E})$ ); it is affine over $S$ and represents the $S$-functor $Y \mapsto \operatorname{Hom}_{\mathcal{O}_{Y}}\left(\mathcal{E}_{(Y)}, \mathcal{O}_{Y}\right)$ [EGAI, 9.4.9].

(b) We assume now that $\mathcal{E}$ is locally free and denote by $\mathcal{E}^{\vee}$ its dual. In this case the affine $S$-scheme $\mathbf{V}(\mathcal{E})$ is of finite presentation (ibid, 9.4.11); also the $S$-functor $Y \mapsto H^{0}\left(Y, \mathcal{E}_{(Y)}\right)=\operatorname{Hom}_{\mathcal{O}_{Y}}\left(\mathcal{O}_{Y}, \mathcal{E}_{(Y)}\right)$ is representable by the affine $S$-scheme $\mathbf{V}\left(\mathcal{E}^{\vee}\right)$ which is also denoted by $\mathbf{W}(\mathcal{E})$ [SGA3, I.4.6].

It applies to the locally free coherent sheaf $\mathcal{E} n d(\mathcal{E})=\mathcal{E}^{\vee} \otimes_{\mathcal{O}_{S}} \mathcal{E}$ over $S$ so that we can consider the affine $S$-scheme $\mathbf{V}(\mathcal{E} n d(\mathcal{E}))$ which is an $S$-functor in associative commutative and unital algebras [EGAI, 9.6.2]. Now we consider the $S$-functor $Y \mapsto \operatorname{Aut}_{\mathcal{O}_{Y}}\left(\mathcal{E}_{(Y)}\right)$. It is representable by an open $S$-subscheme of $\mathbf{V}(\mathcal{E} n d(\mathcal{E}))$ which is denoted by $\mathrm{GL}(\mathcal{E})$ (loc. cit., 9.6.4).

(c) We denote by $\mathbb{Z}[\epsilon]=\mathbb{Z}[x] / x^{2}$ the ring of dual integers and by $S[\epsilon]=S \times_{\mathbb{Z}} \mathbb{Z}[\epsilon]$. If $G / S$ is $S$-group space (i.e. an algebraic space in groups, called group algebraic space over $S$ in [St, Tag043H]) we denote by $\operatorname{Lie}(G)$ the $S$-functor defined by $\operatorname{Lie}(G)(T)=$ $\operatorname{ker}(G(T[\epsilon]) \rightarrow G(T))$. This $S$-functor is a functor in Lie $\mathcal{O}_{S}$-algebras, see [SGA3, II.4.1] or [D-G, II.4.4]. More facts are collected in Appendix 8.2.

(d) If $G / S$ is an affine smooth $S$-group scheme, we denote by $\operatorname{Tors}_{G}(S)$ the groupoid of (right) $G$-torsors over $S$ and by $H^{1}(S, G)$ the set of isomorphism classes of $G$ torsors (locally trivial for the étale topology), we have a classifying map $\operatorname{Tors}_{G}(S) \rightarrow$ $H^{1}(S, G), E \mapsto[E]$.

\section{THE CASE OF TORI}

Lemma 2.1. Let $X$ be a scheme and let $T$ be a $X$-torus. Assume that $T$ is split by a finite étale cover of degree $d$. Then $d H^{1}(X, T) \subseteq H_{Z a r}^{1}(X, T)$.

Proof. Let $f: Y \rightarrow X$ be a finite étale cover of degree $d$ which splits $T$, that is $T_{Y} \cong \mathbb{G}_{m, Y}^{r}$. According to [C-T-S2, 0.4], we have a norm map $f_{*}: H^{1}(Y, T) \rightarrow$ $H^{1}(X, T)$ such that the composite $H^{1}(X, T) \stackrel{f^{*}}{\rightarrow} H^{1}(Y, T) \stackrel{f_{*}}{\rightarrow} H^{1}(X, T)$ is the multiplication by $d$. We claim that $f_{*}\left(H^{1}(Y, T)\right) \subseteq H_{Z a r}^{1}(X, T)$. Let $x \in X$. Then $V_{x}=$ $\operatorname{Spec}\left(\mathcal{O}_{X, x}\right) \times_{X} Y$ is a semi-local scheme so that $\operatorname{Pic}\left(V_{x}\right)=0$ [B:AC, Chap. 2, Sect. 5, No. 3, Proposition 5]. Since $T$ is split over $V_{x}, H^{1}\left(V_{x}, T\right)=0$. Since the construction

of the norm commutes with base change, we get that $\left(f_{*}\left(H^{1}(Y, T)\right)\right)_{\mathcal{O}_{X, x}}=0$. In particular we have $d H^{1}(X, T) \subseteq H_{Z a r}^{1}(X, T)$.

Proposition 2.2. Let $R$ be an henselian local ring of residue field $\kappa$. We denote by $p$ the characteristic exponent of $\kappa$ and let $l$ be a prime number distinct from $p$. Let $X$ be a proper $R$-scheme and let $T$ be a $X$-torus. 
(1) For each $i \geq 0$, the $\operatorname{ker}\left(H^{i}(X, T) \rightarrow H^{i}\left(X_{\kappa}, T\right)\right)$ is l-divisible.

(2) The kernel $\operatorname{ker}\left(H^{0}(X, T) \rightarrow H^{0}\left(X_{\kappa}, T\right)\right)$ is uniquely l-divisible.

(3) We assume that $T$ is locally isotrivial. There exists $r \geq 0$ such that $p^{r} \operatorname{ker}\left(H^{1}(X, T) \rightarrow\right.$ $\left.H^{1}\left(X_{\kappa}, T\right)\right) \subseteq H_{Z a r}^{1}(X, T)$.

Proof. (1) We consider the exact sequence of étale $X$-sheaves $1 \rightarrow{ }_{l} T \rightarrow T \stackrel{\times l}{\longrightarrow} T \rightarrow 1$ which generalizes the Kummer sequence. It gives rise to the following commutative diagram

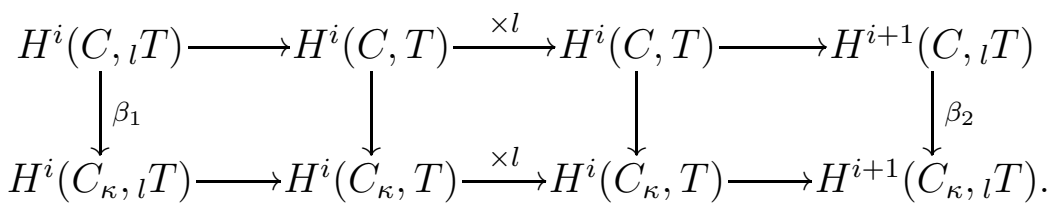

The proper base change theorem [SGA4, XII.5.5.(iii)] shows that $\beta_{1}, \beta_{2}$ are isomorphisms. By diagram chase, we conclude that $\operatorname{ker}\left(H^{i}(C, T) \rightarrow H^{i}\left(C_{\kappa}, T\right)\right)$ is $l$-divisible.

(2) If $i=0$, we can complete the left handside of the diagram with 0. By diagram chase it follows that $\operatorname{ker}\left(H^{0}(C, T) \rightarrow H^{0}\left(C_{\kappa}, T\right)\right)$ is uniquely $l$-divisible.

(3) There exists an open cover $\left(U_{i}\right)_{i=1, \ldots, n}$ of $X$ and finite étale covers $f_{i}: V_{i} \rightarrow U_{i}$ such that $T_{V_{i}}$ is split for $i=1, . ., n$. Let $d$ be the l.c.m. of the degrees of the $f_{i}$ 's. We write $d=p^{r} e$ with $(e, p)=1$. Assertion (1) shows that $\operatorname{ker}\left(H^{1}(X, T) \rightarrow H^{1}\left(X_{\kappa}, T\right)\right)$ is $e$-divisible so that $\operatorname{ker}\left(H^{1}(X, T) \rightarrow H^{1}\left(X_{\kappa}, T\right)\right) \subseteq e H^{1}(X, T)$. Lemma 2.1 shows that $d H^{1}(X, T) \subseteq H_{Z a r}^{1}(X, T)$ which permits to conclude that $p^{r} \operatorname{ker}\left(H^{1}(X, T) \rightarrow\right.$ $\left.H^{1}\left(X_{\kappa}, T\right)\right) \subseteq H_{Z a r}^{1}(X, T)$.

Corollary 2.3. Suppose $T$ quasi-splits after a Galois extension $X^{\prime} / X$ of degree prime to $p$, that is $T \times_{X} X^{\prime} \cong R_{X^{\prime \prime} / X^{\prime}}\left(\mathbb{G}_{m}\right)$ where $X^{\prime \prime} \rightarrow X^{\prime}$ is a finite étale cover. Then $\operatorname{ker}\left(H^{1}(X, T) \rightarrow H^{1}\left(X_{\kappa}, T\right)\right) \subseteq H_{Z a r}^{1}(X, T)$.

Proof. The theorem 90 of Hilbert-Grothendieck shows that $H_{Z a r}^{1}\left(X^{\prime}, T\right)=H^{1}\left(X^{\prime}, T\right)$. By corestriction-restriction it follows that $\left[X^{\prime}: X\right] H^{1}(X, T) \subseteq H_{Z a r}^{1}(X, T)$. In particular we have

$$
\left[X^{\prime}: X\right] \operatorname{ker}\left(H^{1}(X, T) \rightarrow H^{1}\left(X_{\kappa}, T\right)\right) \subseteq H_{Z a r}^{1}(X, T) .
$$

Since $\left[X^{\prime}: X\right]$ is prime with $p$, Proposition 2.2 yields that $\operatorname{ker}\left(H^{1}(X, T) \rightarrow H^{1}\left(X_{\kappa}, T\right)\right) \subseteq$ $H_{Z a r}^{1}(X, T)$.

Corollary 2.4. Suppose char $(k)=0$. Then $\operatorname{ker}\left(H^{1}(X, T) \rightarrow H^{1}\left(X_{\kappa}, T\right)\right) \subseteq H_{Z a r}^{1}(X, T)$. 


\section{Infinitesimal KNESER-Tits PROBLEM}

Let $R$ be a commutative ring and let $G$ be a reductive $R$-group scheme. Let $P$ be a strictly proper $R$-parabolic subgroup of $G$ (it means each projection of $P$ on a semisimple quotient of $G$ is proper) and assume that $P$ admits Levi subgroups. Let $P^{-}$be an opposite $R$-parabolic subgroup to $P$. We denote by $E_{P}(R)$ the subgroup of $G(R)$ which is generated by $\operatorname{rad}(P)(R)$ and $\operatorname{rad}\left(P^{-}\right)(R)$. It does not depend on the choice of $P^{-}[\mathrm{P}-\mathrm{S}, \S 1]$. We denote by $G^{+, P}(R)$ the normal $R$-subgroup of $G(R)$ generated by $\operatorname{rad}(P)(R)$ and $\operatorname{rad}\left(P^{-}\right)(R)$ or equivalently by $E_{P}(R)$. The quotient group $W_{P}(R, G)=G(R) / G^{+, P}(R)$ is called the Whitehead group of $G / R$ with respect to $P$.

Remark 3.1. If $R$ is a field, $E_{P}(R)$ does not depend on the choice of $P$ and the group $E_{P}(R)=G^{+, P}(R)$ is denoted by $G^{+}(R)$ B-T, prop. 6.2]. If for each $s \in \operatorname{Spec}(R)$ each semisimple quotient of $G_{s}$ is of relative rank $\geq 2$, then $E_{P}(R)$ does not depend of the choice of $P$ and $G^{+, P}(R)$ is denoted by $G^{+}(R)$.

The next statements 3.2, 3.5, 3.6 are variations of a result of Borel-Tits on the Whitehead groups over local fields [B-T, prop. 6.14].

Lemma 3.2. Let $U=\operatorname{rad}(P)$ and denote by $U^{\prime} \subset U$ the last $R$-subgroup scheme of Demazure's filtration [SGA3, XXVI.2.1]. Let $s_{1}, \ldots, s_{n}$ be points of $\operatorname{Spec}(R)$ whose residue fields are infinite or finite of characteristic $\neq 2$. We assume that $G$ is semisimple and let $f: G^{s c} \rightarrow G$ be the universal cover. Assume that $\operatorname{ker}(f)$ is smooth.

(1) There exist $g_{1}, \ldots, g_{m} \in G^{+, P}(R)$ such that the product map

$$
h: U^{\prime m} \rightarrow G, \quad\left(u_{1}, \ldots, u_{m}\right) \mapsto{ }^{g_{1}} u_{1} \ldots{ }^{g_{m}} u_{m}
$$

is smooth at $(1, \ldots, 1)_{s_{j}}$ for $j=1, \ldots, n$.

(2) If $R$ is semilocal, the map $d h: \operatorname{Lie}\left(U^{\prime}\right)(R)^{m} \rightarrow \operatorname{Lie}(G)$ is onto.

This requires a variation on a statement of Riehm on proper subalgebras of Chevalley Lie algebras [R, §2, Lemma].

Lemma 3.3. Let $G$ be a semisimple group defined over a field $F$.

(1) Suppose that the characteristic of $F$ is not 2 and that $G$ is split and almost simple. Let $L$ be a Lie subalgebra of $\operatorname{Lie}(G)$ which is $G(F)$-stable and contains a long root element (i.e. associated to some long root). Then $L=\operatorname{Lie}(G)$.

(2) Suppose that $G$ is simply connected. Let $P$ be a strictly proper parabolic subgroup of $G$ and put $U=\operatorname{rad}(P)$. We denote by $U^{\prime} \subset U$ the last $F$-subgroup of Demazure's filtration [SGA3, XXVI.1.2], which is a vector F-group scheme.

Then $\operatorname{Lie}(G)$ is the unique Lie $F$-subalgebra of $\operatorname{Lie}(G)$ containing $\operatorname{Lie}\left(U^{\prime}\right)$ and which is stable under the adjoint action of $G$. 
Proof. (1) Since roots of maximal length are conjugated under the Weyl group and since maximal split tori of $G$ are $G(F)$-conjugated, it follows that all long root elements are $G(F)$-conjugated. It follows that $L$ contains all long root elements. According to [C-S-U-W, prop. 3.3], we conclude that $L=\operatorname{Lie}(G)$.

(2) Without loss of generality we can assume that $F$ is algebraically closed and that $G$ is almost simple. Let $B$ be a Borel subgroup of $P$ and let $T$ be a maximal $F$-torus of $B$. Let $U_{\max }$ be the root subgroup attached to the maximal root of $\Phi(G, T)$ for the order defined by $B$. Then $U_{\max } \subset U^{\prime}$. Now let $L$ be a Lie subalgebra of $\operatorname{Lie}(G)$ which contains $\operatorname{Lie}\left(U^{\prime}\right)$ and is stable under the adjoint action of $G$. If $\operatorname{char}(\mathrm{k}) \neq 2$ statement (1) shows that $L=\operatorname{Lie}(G)$ since $L$ contains $\operatorname{Lie}\left(U_{\max }\right)$.

It remains to discuss the characteristic two case. The argument of (1) shows that $L$ contains all long root elements. The proper $G$-submodules of $\operatorname{Lie}(G)$ are listed in C-G-P, lemma 7.1.2]. Assume that $L \subsetneq \mathfrak{g}=\operatorname{Lie}(G)$. Let $\mathfrak{t}$ be a Cartan subalgebra of $\mathfrak{g}=\operatorname{Lie}(G)$, up to conjugacy, we have that $L \subset \mathfrak{t} \oplus \mathfrak{g}_{<}$where $\mathfrak{g}_{<}$is generated by eigenspaces attached to short roots. Then $\mathfrak{g}_{>} \cap L=0$ which contradicts the fact that $L$ contains all long root elements.

We proceed now to the proof of Lemma 3.2 .

Proof. Without loss of generality, we can assume that $G$ and $P$ are of constant type. The hypothesis implies that $G^{s c} \rightarrow G$ is étale and then reduces to the simply connected case. Let $s_{j}$ be one of the point.

Case $\kappa\left(s_{j}\right)$-infinite. According to Lemma 3.3. (2), Lie $(G)\left(\kappa\left(s_{j}\right)\right)$ is the only subspace of $\operatorname{Lie}(G)\left(\kappa\left(s_{j}\right)\right)$ containing $\operatorname{Lie}\left(U^{\prime}\right)\left(\kappa\left(s_{j}\right)\right)$ and stable under the adjoint action of $G_{\kappa\left(s_{j}\right)}$. Since $G^{+}\left(\kappa\left(s_{j}\right)\right)$ is Zariski dense in $G_{\kappa\left(s_{j}\right)}$, it follows that there exists $g_{1, j}, \ldots$, $g_{m_{j}, j} \in G^{+}\left(\kappa\left(s_{j}\right)\right)$ such that $\operatorname{Lie}(G)\left(\kappa\left(s_{j}\right)\right)$ is generated by the ${ }^{g_{i, j}} \operatorname{Lie}\left(U^{\prime}\right)\left(\kappa\left(s_{j}\right)\right)$.

Case $\kappa\left(s_{j}\right)$ finite. According to Lemma 3.3. (1), Lie $(G)\left(\kappa\left(s_{j}\right)\right)$ is the only subspace of $\operatorname{Lie}(G)\left(\kappa\left(s_{j}\right)\right)$ containing Lie $\left(U^{\prime}\right)\left(\kappa\left(s_{j}\right)\right)$ and stable under $G\left(\kappa\left(s_{j}\right)\right)$. The group $G_{\kappa\left(s_{j}\right)}$ is quasi-split so that $G^{+}\left(\kappa\left(s_{j}\right)\right)=G\left(\kappa\left(s_{j}\right)\right)$ [T, $\left.\S 1.1 .2\right]$; it follows that there exists $g_{1, j}$, $\ldots, g_{m_{j}, j} \in G^{+}\left(\kappa\left(s_{j}\right)\right)$ such that $\operatorname{Lie}(G)\left(\kappa\left(s_{j}\right)\right)$ is generated by the ${ }^{g_{i, j}} \operatorname{Lie}\left(U^{\prime}\right)\left(\kappa\left(s_{j}\right)\right)$.

We gather now both cases.

Claim 3.4. The map $G^{+, P}(R) \rightarrow \prod_{j} G^{+, P}\left(\kappa\left(s_{j}\right)\right)$ is onto.

Let $P^{-}$be an opposite parabolic subgroup scheme of $P$ and let $U^{-}$be its unipotent radical. Since $G^{+, P}(R)$ (resp. each $G^{+, P}\left(\kappa\left(s_{j}\right)\right)$ ) is generated by $U(R)$ and $U^{-}(R)$, it is enough to show the surjectivity of $U(R) \rightarrow \prod_{j} U\left(\kappa\left(s_{j}\right)\right)$. According to [SGA3, XXVI.2.5], there exists a f.g. locally free $R$-module $\mathcal{E}$ such that $U$ is isomorphic to $\mathbf{W}(\mathcal{E})$ as $R$-scheme. Since $\mathbf{W}(\mathcal{E})(R)=\mathcal{E}$ maps onto $\prod_{j} \mathbf{W}(\mathcal{E})\left(\kappa\left(s_{j}\right)\right)=\prod_{j} \mathcal{E} \otimes_{R} \kappa\left(s_{j}\right)$, the Claim is established. 
There are $g_{1}, \ldots, g_{m} \in G(R)$ such that $\operatorname{Lie}(G)\left(\kappa\left(s_{j}\right)\right)$ is generated by the ${ }^{g_{i}} \operatorname{Lie}\left(U^{\prime}\right)\left(\kappa\left(s_{j}\right)\right)$ for $j=1, \ldots, n$. The differential of the product map

$$
h: U^{\prime m} \rightarrow G, \quad\left(u_{1}, \ldots, u_{m}\right) \mapsto{ }^{g_{1}} u_{1} \ldots{ }^{g_{m}} u_{m}
$$

is $\operatorname{Lie}\left(U^{\prime}\right)^{m} \rightarrow \operatorname{Lie}(G), \quad\left(x_{1}, \ldots, x_{m}\right) \mapsto{ }^{g_{1}} x_{1} \ldots{ }^{g_{m}} x_{m}$. It is onto by construction and we conclude that $h$ is smooth at $(1, \ldots, 1)_{s_{j}}$ for $j=1, \ldots, n$.

(2) We assume that $R$ is semilocal with maximal ideals $\mathfrak{m}_{1}, \ldots, \mathfrak{m}_{t}$. Then $J=\mathfrak{m}_{1} \cap$ $\cdots \cap \mathfrak{m}_{t}$ is the Jacobson radical of $R$ and $R / J \cong R / \mathfrak{m}_{1} \times \ldots R / \mathfrak{m}_{t}$. Statement (1) shows that the map $d h: \operatorname{Lie}\left(U^{\prime}\right)(R)^{m} \rightarrow \operatorname{Lie}(G)(R)$ is surjective modulo $\mathfrak{m}_{i}$ for $i=1, \ldots, t$ so is surjective modulo $J$. Since $\operatorname{Lie}(G)(R)$ is finitely generated, Nakayama's lemma $[\mathrm{Ks}$, II.4.2.3] enables us to conlude that $d h$ is onto.

The two next statements will not be used in the paper but are applications of Lemma 3.2 to the rigidity of Whitehead groups. Let $I$ be an ideal of $R$ satisfying $I^{2}=0$ and consider the exact sequence ([D-G, proof of II.5.2.8] or Lemma 8.3. $(2)$ )

$$
1 \rightarrow \operatorname{Lie}(G)(R) \otimes_{R} I \stackrel{\mathrm{e}^{?}}{\rightarrow} G(R) \rightarrow G(R / I) \rightarrow 1 .
$$

Lemma 3.5. The sequence

$$
\operatorname{Lie}(G)(R) \otimes_{R} I \rightarrow G(R) / G^{+, P}(R) \rightarrow G(R / I) / G^{+, P}(R / I) \rightarrow 1
$$

is exact.

Proof. We are given an element $g \in G(R)$ whose reduction in $G(R / I)$ belongs to $G^{+, P}(R / I)$. Since the maps $G(R) \rightarrow G(R / I)$ and $\operatorname{rad}\left(P^{ \pm}\right)(R) \rightarrow \operatorname{rad}\left(P^{ \pm}\right)(R / I)$ are onto, it follows that $G^{+, P}(R) \rightarrow G^{+, P}(R / I)$ is onto. Therefore we have $g \in$ $\mathrm{e}^{\left(\operatorname{Lie}(G)(R) \otimes_{R} I\right)} G^{+, P}(R)$. This shows the exactness.

Proposition 3.6. We assume that $R$ is semilocal with infinite residue fields and $I$ an ideal of $R$ with $I^{d}=0$ for some $d \geq 2$. Assume that $G$ is semisimple and let $f: G^{\text {sc }} \rightarrow G$ be the universal cover. Assume that $\operatorname{ker}(f)$ is smooth. The map $G(R) / G^{+, P}(R) \rightarrow G(R / I) / G^{+, P}(R / I)$ is an isomorphism.

Proof. Induction on $d$ reduces to the case $d=2$. Let $s_{1}, \ldots, s_{n}$ be the closed points of $\operatorname{Spec}(R)$. We have to show the inclusion $\mathrm{e}^{\left(\operatorname{Lie}(G)(R) \otimes_{R} I\right)} \subset G^{+, P}(R)$. Lemma 3.2 provides elements $g_{1}, \ldots, g_{m} \in G(R)$ such that the product map $h: U^{\prime} m \rightarrow$ $G,\left(u_{1}, \ldots, u_{l}\right) \mapsto{ }^{g_{1}} u_{1} \ldots{ }^{g} u_{m}$ is smooth at $(1, \ldots, 1)_{s_{j}}$ for $j=1, \ldots, n$. Nakayama lemma shows that $d h: \operatorname{Lie}\left(U^{\prime}\right)(R)^{m} \rightarrow \operatorname{Lie}(G)(R)$ is onto. By construction we have $\mathrm{e}^{\left(d h\left(\operatorname{Lie}\left(U^{\prime}\right)(R)^{m} \otimes_{R} I\right)\right)} \subseteq G^{+, P}(R)$. Thus e $\mathrm{e}^{\left(\operatorname{Lie}(G)(R) \otimes_{R} I\right)} \subseteq G^{+, P}(R)$ as desired. 


\section{MOduli STACK OF $G$-TORSORS}

4.1. Setting. Let $S$ be a noetherian separated base scheme. Let $p: C \rightarrow S$ be a projective flat relative curve (that is all geometric fibers are algebraic curves). We assume that $C$ is integral and that the map $\mathcal{O}_{S} \rightarrow p_{*}\left(\mathcal{O}_{C}\right)$ is universally an isomorphism. This implies that $p$ is cohomologically flat in dimension zero, that the formation of $p_{*} \mathcal{O}_{C}$ commutes with base change. We recall that $p$ is cohomologically flat in degree 1 for each $S$-flat coherent sheaf $\mathcal{F}$ over $C$ [I, 8.3.11.1]; it means that the formation of $R^{1} p_{*} \mathcal{F}$ commutes with base change.

Let $G$ be a smooth affine group scheme over $C$. We assume that $G$ admits a linear representation $i: G \rightarrow \operatorname{GL}(\mathcal{E})$ where $\mathcal{E}$ is a locally free coherent sheaf of constant rank such that

(I) $i$ is a closed immersion;

(II) the fppf sheaf $\mathrm{GL}(\mathcal{E}) / G$ is representable by an affine $C$-scheme.

Note that the smoothness of $\operatorname{GL}(\mathcal{E})$ implies that $\operatorname{GL}(\mathcal{E}) / G$ is smooth SGA3, $\left.\mathrm{VI}_{B} \cdot 9.2\right]$. Also this assumption is satisfied for example if $G$ is a semisimple group scheme [Ma, prop. 3.2].

Lemma 4.1. Let $Y$ be an $S$-scheme and let $P, Q$ be two $G$-torsors over $C_{Y}$. Then the fppf $Y$-sheaf

$$
Y^{\prime} \mapsto \operatorname{Isom}_{G_{C_{Y^{\prime}}}}\left(P_{C_{Y^{\prime}}}, Q_{C_{Y^{\prime}}}\right)
$$

is representable by a $Y$-scheme $\operatorname{Isom}^{b}(P, Q)$ which is affine of finite presentation.

Proof. Since the $G_{Y}$-torsors $P$ and $Q$ are locally isomorphic over $C_{Y}$ to $G_{C_{Y}}$ with respect to the étale topology, the faithfully flat descent theorem shows that the $C_{Y^{-}}$ functor

$$
D \mapsto \operatorname{Isom}_{G \times_{C} D}\left(P \times_{C_{Y}} D, Q \times_{C_{Y}} D\right)
$$

is representable by an affine smooth $C_{Y}$-scheme $\operatorname{Isom}_{G}(P, Q)$. The $Y$-functor

$$
Y^{\prime} \mapsto \operatorname{Isom}_{G_{C_{Y^{\prime}}}}\left(P_{C_{Y^{\prime}}}, Q_{C_{Y^{\prime}}}\right)
$$

is nothing but $\prod_{C_{Y} / Y}\left(\mathbf{I s o m}_{G}(P, Q) / C_{Y}\right)$. According to Grothendieck [FGA, TDTE, $\S \mathrm{C} .2]$, the functor $\prod_{C_{Y} / Y}\left(\operatorname{Isom}_{G}(P, Q) / C_{Y}\right)$ is representable by a $Y$-scheme. We denote by $\operatorname{Isom}^{b}(P, Q)$ the relevant $Y$-scheme. It is actually affine over $Y$ by the argument of [Hd1, §1.4]. Since the global section functor is locally of finite presentation, we have that $\operatorname{Isom}^{b}(P, Q)$ is of finite presentation.

For each $S$-scheme $Y$, we denote by $\operatorname{Bun}_{G}(Y)=\operatorname{Tors}_{G}\left(C_{Y}\right)$ the groupoid of $C_{Y^{-}}$ torsors under $G_{C_{Y}}$. This defines a $S$-stack. The following extends partially [He1, prop. 1]. It is based on the crucial case of $\mathrm{GL}_{n}$ and use of a dévissage method due to Sorger [So, §3.6]. 
Proposition 4.2. The S-stack $\operatorname{Bun}_{G}$ is a smooth (quasi-separated) algebraic stack locally of finite type.

Proof. We shall firstly establish that the stack $\operatorname{Bun}_{G}$ is algebraic. In the $\mathbf{G L}_{n}$-case, the stack Bun $\mathrm{GL}_{n}$ is that of locally free coherent modules of rank $n$ denoted by $\mathcal{F} i b_{C / S, n}$. It is algebraic locally of finite type [L-M-B, th. 4.6.21].

The next case is that of $\mathrm{GL}(\mathcal{E})$ for $\mathcal{E}$ locally free of rank $n$. We denote by $P=$ $\underline{\operatorname{Isom}}\left(\mathcal{O}_{C}^{n}, \mathcal{E}\right)$ the associated $\mathrm{GL}_{n}$-torsor over $C$. Since $\mathrm{GL}(\mathcal{E})=P \wedge \mathrm{GL}_{n} \mathrm{GL}_{n}$ is the twist of $\mathrm{GL}_{n}$ by $P$, twisting by $P$ provides an isomorphism of stacks $\mathrm{Bun}_{\mathrm{GL}_{n}} \rightarrow \mathrm{Bun}_{G}$, so that $\operatorname{Bun}_{G}$ is an algebraic stack locally of finite type according to the preceding case. For the general case, the representation $i: G \rightarrow \mathrm{GL}(\mathcal{E})$ gives rise to a 1-morphism of $S-\operatorname{stacks} \varphi: \operatorname{Bun}_{G} \rightarrow \operatorname{Bun}_{\mathrm{GL}(\mathcal{E})}$.

Claim 4.3. The morphism $\varphi$ is representable.

We are given a $S$-scheme $U$ and a morphism $\eta: U \rightarrow \operatorname{Bun}_{\mathrm{GL}(\mathcal{E})}$, that is a $\operatorname{GL}(\mathcal{E})-$ bundle $F_{\eta}$ over $C_{U}$. We consider the $S$-stack $B_{\eta}=U \times_{\mathrm{Bun}_{\mathrm{GL}(\mathcal{E})}} \operatorname{Bun}_{G}$. On the other hand, we consider the fppf sheaf $M_{\eta}=F_{\eta} \wedge(\mathrm{GL}(\mathcal{E}) / G)$; it is representable by an affine $U$-scheme of finite presentation by faithfully flat descent. We denote by $q: F_{\eta} \rightarrow M_{\eta}$ the quotient map, this is a $G$-torsor.

Let $V$ be a $U$-scheme. Then $B_{\eta}(V)$ is the groupoid of pairs $(P, \alpha)$ where $P$ is a $G$-torsor over $V$ and $\alpha: P \wedge^{G} \operatorname{GL}(\mathcal{E}) \stackrel{\sim}{\longrightarrow} F_{\eta, V}$ is an isomorphism of $\operatorname{GL}(\mathcal{E})$-torsors.

Each point $m \in M_{\eta}\left(C_{V}\right)$ defines the $G$-torsor $P_{m}=q^{-1}(m)$ and a trivialization $\alpha_{m}: P_{m} \wedge^{G} \operatorname{GL}(\mathcal{E}) \stackrel{\sim}{\longrightarrow} F_{\eta, V}$ given by $(p, f) \mapsto i_{m}(p) f$ (where $i_{m}: P_{m} \rightarrow F_{\eta, V}$ is the obvious embedding). We get then a morphism of $U$-stacks $j: \prod_{C_{U} / U} M_{\eta} \rightarrow B_{\eta}$. The point is that the $U$-functor $\prod_{C_{U} / U} M_{\eta}$ is representable by a $V$-scheme [FGA, TDTE, §C.2] which is affine of finite presentation. Since the groupoid $\prod_{C_{U} / U}\left(M_{\eta}\right)$ is equivalent to $B_{\eta}(V)$ for each $V$ [Gd, III.2.1], we have shown that $\varphi$ is representable.

According to [St, 86.15.4, Tag 05UM], the Claim implies that $\operatorname{Bun}_{G}$ is an algebraic stack. To show the smoothness, we can use the criterion of formal smoothness [He2, 2.6] (or [St, §98.8, Tag 0DNV]). We are given a $S$-ring $R$ which is local Artinian with maximal ideal $\mathfrak{m}$ such that $\mathfrak{m}^{2}=0$ and a $G$-torsor $P_{0}$ over $C_{0}=C \times_{S} R / \mathfrak{m}$. We put $G_{0}=G_{C_{0}}$ and denote by $H_{0}=P_{0} \wedge^{G_{0}} G_{0}$ the twisted group scheme over $C_{0}$. According to [I, th. 2.6], the obstruction to lift $P_{0}$ in a $G$-torsor over $C \times{ }_{S} R$ is a class of $H^{2}\left(C_{0}, \mathcal{L} i e\left(H_{0}\right) \otimes_{\mathcal{O}_{C_{0}}} \mathfrak{m}\right)$. But $R / \mathfrak{m}=\kappa$ is a field and $C_{0}$ is of dimension 1 so that this group vanishes according to Grothendieck's vanishing theorem [Ha, III.2.7]. The formal smoothness criterion is satisfied so that the algebraic stack Bun Bu $_{G}$ ismooth.

It remains to show that $\operatorname{Bun}_{G}$ is locally of finite type. We take an atlas $\eta: U \rightarrow$ $\operatorname{Bun}_{\mathrm{GL}(\mathcal{E})}$ where $U$ is smooth locally of finite type (and quasi-separated). Since $B_{\eta} \rightarrow$ $\operatorname{Bun}_{G}$ is an atlas and $B_{\eta} \rightarrow U$ is affine of finite presentation, $B_{\eta}$ is locally of finite type over $S$ (and quasi-separated). We conclude that $\operatorname{Bun}_{G}$ is locally of finite type. 
4.2. The tangent stack. We consider now the tangent stack $T\left(\operatorname{Bun}_{G} / S\right)[\mathrm{L}-\mathrm{M}-\mathrm{B}$, §17] which is algebraic (loc. cit., 17.16). By definition, for each $S$-scheme $Y$, we have $T\left(\operatorname{Bun}_{G} / S\right)(Y)=\operatorname{Bun}_{G}(Y[\epsilon])$ where $\left.Y[\epsilon]\right)=Y \times_{\mathbb{Z}} \mathbb{Z}[\epsilon]$. It comes with two 1 -morphisms

$$
\tau: T\left(\operatorname{Bun}_{G} / S\right) \rightarrow \operatorname{Bun}_{G}
$$

and $\sigma: \operatorname{Bun}_{G} \rightarrow T\left(\operatorname{Bun}_{G} / S\right)$.

Remark 4.4. We can consider the smooth-étale site on $\mathrm{Bun}_{G}$ and the quasi-coherent sheaf $\Omega_{\mathrm{Bun}_{G} / S}^{1}$; its associated generalized vector bundle $\mathbb{V}\left(\Omega_{\mathrm{Bun}_{G} / S}^{1}\right)$ is an algebraic $S$ stack. There is a canonical 1-isomorphism between $T\left(\operatorname{Bun}_{G} / S\right)$ and $\mathbb{V}\left(\Omega_{\mathrm{Bun}_{G} / S}^{1}\right)$ (loc. cit., 17.15). We shall not use that fact in the paper.

Our goal is the understanding of the fiber product of $S$-stacks

$$
\mathcal{T}_{b}=T\left(\operatorname{Bun}_{G} / S\right) \times_{\operatorname{Bun}_{G}} S
$$

where $b: S \rightarrow \operatorname{Bun}_{G}$ corresponds to the trivial $G$-bundle $G$ over $C$. According to [L-M-B, 2.2.2], for each $S$-ring $B$, the fiber category $\mathcal{T}_{b}(B)$ has for objects the couples $(F, f)$ where $F$ is a $G_{C_{B}}[\epsilon]$-torsor and $f: G_{C_{B}} \stackrel{\sim}{\longrightarrow} F \times_{C_{B[\epsilon]}} C_{B}$ is a trivialization of $G_{C_{B}}$-torsors; an arrow $\left(F_{1}, f_{1}\right) \rightarrow\left(F_{2}, f_{2}\right)$ is a couple $(H, h)$ where $H$ : $F_{1} \stackrel{\sim}{\longrightarrow} F_{2}$ is an isomorphism of $G_{C_{B[\epsilon]}}$-torsors and $h \in G\left(C_{B}\right)$ with the compatibility $\left(H \times_{C_{B[\epsilon]}} C_{B}\right) \circ f_{1}=f_{2} \circ h$.

4.3. Relation with the Lie algebra. We consider the Weil restriction $G^{\prime}=\prod_{C[\epsilon] / C} G$, this is an affine smooth $C$-group scheme [C-G-P, A.5.2]. It comes with a $C$-group homomorphism $j: G \rightarrow G^{\prime}$ and with a $C[\epsilon]$-group homomorphism $q: G^{\prime} \times_{C} C[\epsilon] \rightarrow$ $G \times{ }_{S} S[\epsilon]$.

We consider the functor $\Phi$ between the categories of $G^{\prime}$-torsors over $C$ and that of $G$-torsors over $C[\epsilon]$ defined by the assignment $E^{\prime} \mapsto q_{*}\left(E^{\prime} \times_{C} C[\epsilon]\right)$.

For each affine open subset $V$ of $C$, the map $H^{1}\left(V, G^{\prime}\right) \rightarrow H^{1}(V[\epsilon], G)$ is bijective Gd, VII.1.3.2] so that each $G$-torsor over $C[\epsilon]$ is trivialized by an étale cover of $C$ extended to $C[\epsilon]$. According to [SGA3, XXIV.8.2] (see also [Gd, III.3.1.1]), it folllows that we can define the functor $\Psi$ by the assignment $F^{\prime} \mapsto \prod_{C[\epsilon] / C}\left(F^{\prime}\right)$. The functors $\Phi$ and $\Psi$ are inverse of each others so that the groupoids $\operatorname{Tors}_{G^{\prime}}(C)$ and $\operatorname{Tors}_{G \times} C[\epsilon](C[\epsilon])$ are isomorphic. We come now to Lie algebra considerations. By definition of the Lie algebra, the $C$-group $G^{\prime}$ fits in an exact sequence of $C$-group schemes

$$
0 \rightarrow \mathbf{W}(\mathcal{L} i e(G)) \stackrel{\mathrm{e}^{?}}{\rightarrow} G^{\prime} \stackrel{\pi}{\rightarrow} G \rightarrow 1
$$

where $\operatorname{Lie}(G)=\omega_{G / S}^{\vee}$ (see $\$ 8.2$ and Remark 8.4.(a)). According to [Gd, III.3.2.1] we have an equivalence of groupoids between $\operatorname{Tors}_{\mathbf{W}}(\mathcal{L} i e(G))(C)$ and that of couples $\left(E^{\prime}, \eta\right)$ where $E^{\prime}$ is a $G^{\prime}$-torsor over $C$ and $\eta: G \stackrel{\sim}{\longrightarrow} \pi_{*} E^{\prime}$ is a trivialization. Taking into account the previous isomorphism of categories, we get then an equivalence of 
groupoids between $\operatorname{Tors}_{\mathbf{W}(\mathcal{L} i e(G))}(C)$ and that of couples $(F, \xi)$ where $F$ is a $G$-torsor over $C[\epsilon]$ and $\xi: G_{C} \rightarrow F \times_{C} C[\epsilon]$ is a trivialization; the morphisms are clear.

We come back now to the previous section involving a $S$-ring $R$ and the morphism $b: \operatorname{Spec}(R) \rightarrow \operatorname{Bun}_{G}$ associated to the trivial $G$-torsor. By comparison it follows that the fiber category $\mathcal{T}_{b}(R)$ is equivalent to $\operatorname{Tors}_{\mathbf{W}}(\mathcal{L} i e(G))(C)$.

\section{UNIFORMIZATION AND LOCAL TRIVIALITY}

5.1. Loop groups. We continue with the framework of the previous section and assume from now on for simplicity that $S=\operatorname{Spec}(R)$ is affine noetherian. We deal with a projective flat relative curve $p: C \rightarrow \operatorname{Spec}(R)$, such that $C$ is integral and such that the map $\mathcal{O}_{S} \rightarrow p_{*}\left(\mathcal{O}_{C}\right)$ is universally an isomorphism.

Examples 5.1. We assume all previous assumptions on $C \rightarrow \operatorname{Spec}(R)$ except the last one and discuss the map $\mathcal{O}_{S} \rightarrow p_{*}\left(\mathcal{O}_{C}\right)$.

(a) If $R$ is noetherian local, and the geometric fibers of $C$ are reduced and connected, then $p$ is cohomologically flat in degree zero and the map $\mathcal{O}_{S} \rightarrow p_{*}\left(\mathcal{O}_{C}\right)$ is an isomorphism [EGAIII, 7.8.6], see also [I, Prop. 8.5.16]. Since the formation of $p_{*}\left(\mathcal{O}_{C}\right)$ commutes with base change, we conclude that the map $\mathcal{O}_{S} \rightarrow p_{*}\left(\mathcal{O}_{C}\right)$ is an universal isomorphism.

(b) We assume in the sequel that $R$ is a DVR with fraction field $K$ and residue field $\kappa$.

(b1) If $C_{K}$ is furthermore geometrically integral, the map $\mathcal{O}_{S} \rightarrow p_{*}\left(\mathcal{O}_{C}\right)$ is an isomorphism [L, 8.3.6].

(b2) We assume furthermore that the scheme $C$ is normal, satisfies $\mathcal{O}_{S} \stackrel{\sim}{\longrightarrow} p_{*}\left(\mathcal{O}_{C}\right)$ and that the g.c.d. of the geometric multiplicites of the irreducible components $C_{\kappa}$ is prime to the characteristic exponent of $\kappa$. Then $C / R$ is cohomologically flat in dimension zero [Ra, Introduction].

(b3) We assume that furthermore $C$ is normal, that $C_{K}$ is geometrically integral, the g.c.d. of the geometric multiplicities of the irreducible components $C_{\kappa}$ is prime to the characteristic exponent of $\kappa$. By combining (b1) and (b2), we get that $\mathcal{O}_{S} \stackrel{\sim}{\longrightarrow} p_{*}\left(\mathcal{O}_{C}\right)$ and that $C / R$ is cohomologically flat in dimension zero.

We are given a finite $S$-scheme $D$ with a closed embedding $s: D \rightarrow C$ such that

(i) $D$ is an effective Cartier divisor which is ample;

(ii) $s$ factorizes through an affine $R$-subcheme $V$ of $C$.

Note that (i) implies that the open complement $V^{\prime}=C \backslash D$ is affine over $S$. Let $V=\operatorname{Spec}(A), V^{\prime}=\operatorname{Spec}\left(A^{\prime}\right)$ and $V \cap V^{\prime}=\operatorname{Spec}\left(A_{\sharp}\right)$; this intersection is affine because the morphism $C \rightarrow S$ is a separated [St, 25.21.7.(1), Tag 01KP]. We denote by $I \subset A$ the ideal defining $D$. We consider the completed ring $\widehat{A}:=\widehat{A}_{I}=\varliminf_{n} A / I^{n}$. We need some basic facts from commutative algebra (see [B:AC, III.4.3, th. 3 and prop. 8] for (a) and (b)). 
(a) $\widehat{A}$ is noetherian and flat over $A$.

(b) The assignment $\mathfrak{m} \mapsto \mathfrak{m} \widehat{A}$ provides a correspondence between the maximal ideals of $A$ containing $I$ and the maximal ideals of $\widehat{A}$;

(c) If $R$ is semilocal so is $\widehat{A}$.

If $R$ is local, the finite $R$-algebra $A / I$ is semilocal so we get (c) from (b).

We recall that $G$ is a smooth affine group scheme over $C$, it admits a linear representation $i: G \rightarrow \operatorname{GL}(\mathcal{E})$ where $\mathcal{E}$ is a locally free coherent sheaf of constant rank such that $i$ is a closed immersion and such that the fppf sheaf $\mathrm{GL}(\mathcal{E}) / G$ is representable by an affine $C$-scheme. We consider the following $R$-functors defined for each $R$-algebra $B$ by:

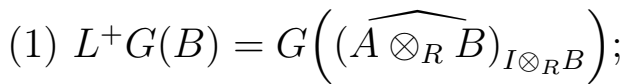

(2) $L G(B)=G\left(\left(\widehat{A \otimes_{R} B}\right)_{I \otimes B} \otimes_{A} A_{\sharp}\right)$.

Example 5.2. (a) The simplest example of our situation is $C=\mathbb{P}_{R}^{1}=V^{\prime} \cup V=$ $\operatorname{Spec}(R[t]) \cup \operatorname{Spec}\left(R\left[t^{-1}\right]\right)$ and for $D$ the point 0 of $C$. In this case, we have $A=R[t]$, $I=t A, A_{\sharp}=R\left[t, t^{-1}\right]$ and $\widehat{A}=R[[t]]$. For each $R$-algebra $B$, we have $\left(\widehat{A \otimes_{R} B}\right)_{I \otimes B}=$ $B[[t]]$ and $\left(\widehat{A \otimes_{R} B}\right)_{I \otimes_{R} B} \otimes_{A} A_{\sharp}=B[[t]] \otimes_{R[t]} R\left[t, t^{-1}\right]=B[[t]]\left[\frac{1}{t}\right]$. The standard notation for the last ring is $B((t))$.

5.2. Patching. For simplicity we assume that $S=\operatorname{Spec}(R)$ where $R$ is a noetherian ring. If we are given a $R$-ring $B$ (not necessarily noetherian), we need to deal with the rings $\left(\widehat{A \otimes_{R} B}\right)_{I \otimes B}$ and $\left(\widehat{A \otimes_{R} B}\right)_{I \otimes B} \otimes_{A} A_{\sharp}$. As pointed out by Bhatt [Bh, $\left.\S 1.3\right]$, the Beauville-Laszlo theorem [B-L] states that one can patch compatible quasi-coherent sheaves on $\operatorname{Spec}\left(\left(\widehat{A \otimes_{R} B}\right)_{I \otimes B}\right)$ and $V_{B}^{\prime}$ to a quasi-coherent sheaf on $C_{B}$, provided the sheaves being patched are flat along $\operatorname{Spec}(B / I B)$. In particular the square of functors

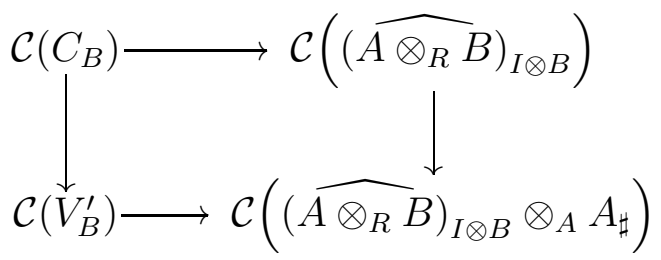

is cartesian where $\mathcal{C}(X)$ stands for the category of flat quasi-coherent sheaves over the scheme $X$ (resp. the category of flat affine schemes over $X$ ). Note that if the ring $B$ is noetherian, Ferrand-Raynaud's patching [F-R] (see also [M-B]) does the job. 
Proposition 5.3. (1) The square of functors

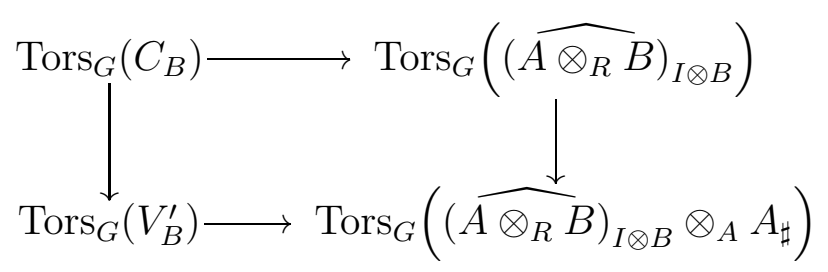

is cartesian.

(2) The $S$-functor $L G$ is isomorphic to the functor associating to each $R$-ring $B$ the $G$-torsors over $C_{B}$ together with trivializations on $V_{B}^{\prime}$ and on $\operatorname{Spec}\left(\left(\widehat{A \otimes_{R} B}\right)_{I \otimes B}\right)$.

Proof. (1) Since $G$ is affine and flat over $C$, it is a formal corollary of the patching statement.

(2) Let $\mathcal{C}(B)$ be the the category of $G$-torsors over $C_{B}$ together with trivializations on $V_{B}^{\prime}$ and on $\operatorname{Spec}\left(\left(\widehat{A \otimes_{R} B}\right)_{I \otimes B}\right)$. An object of $\mathcal{C}(B)$ is a triple $\left(E, f_{1}, f_{2}\right)$ where $E$ is a $G_{C_{B}}$-torsor, $f_{1}: G_{V_{B}^{\prime}} \stackrel{\sim}{\longrightarrow} E_{V_{B}^{\prime}}$ and $f_{2}: G_{\left(\widehat{A \otimes_{R} B}\right)_{I \otimes B}} \stackrel{\sim}{\longrightarrow} E_{\left(\widehat{A \otimes_{R} B}\right)_{I \otimes B}}$ are trivializations. An element $g \in L G(B)=G\left(\left(\widehat{A \otimes_{R} B}\right)_{I \otimes B} \otimes_{A} A_{\sharp}\right)$ gives rise to the right translation

$$
\left(G_{V_{B}^{\prime}}\right)_{\left(\widehat{A \otimes_{R} B}\right)_{I \otimes B} \otimes_{A} A_{\sharp}} \stackrel{\sim}{\longrightarrow}\left(G_{(\widehat{A \otimes R} B)_{I \otimes B}}\right)_{\left(\widehat{A \otimes_{R} B}\right)_{I \otimes B} \otimes_{A} A_{\sharp}} .
$$

It defines a $G_{C}$-torsor $E_{g}$ with trivializations $f_{1}$ and $f_{2}$ on $V_{B}^{\prime}$ and on $\operatorname{Spec}\left(\left(\widehat{A \otimes_{R} B}\right)_{I \otimes B}\right)$. We get then a morphism $\Phi: L G(B) \rightarrow \mathcal{C}(B)$.

Conversely let $c=\left(T, f_{1}, f_{2}\right)$ be an object of $\mathcal{C}(B)$. Then the map $f_{1}^{-1} f_{2}$ : $G_{\left(\widehat{A \otimes_{R} B}\right)_{I \otimes B} \otimes_{A} A_{\sharp}} \rightarrow G_{\left(\widehat{A \otimes_{R} B}\right)_{I \otimes B} \otimes_{A} A_{\sharp}}$ is an isomorphism of $G$-torsors hence is the right translation by an element $g=\Psi(c) \in G\left(\left(\widehat{A \otimes_{R} B}\right)_{I \otimes B} \otimes_{A} A_{\sharp}\right)$. The functors $\Phi$ and $\Psi$ provide the desired isomorphism of functors.

Continuing with the $R$-ring $B$, we have a factorization

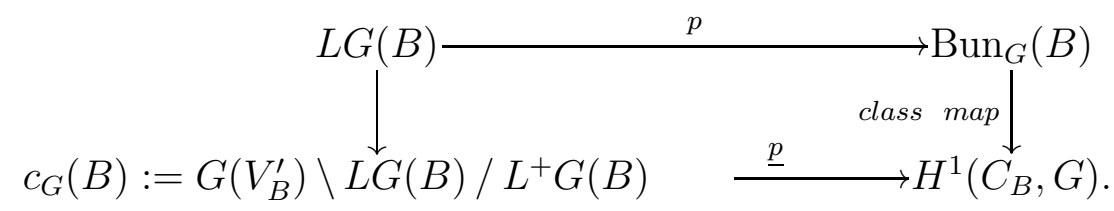

The map $p$ is called the uniformization map. Proposition 5.3. (2) implies that the bottom map induces a bijection

$$
c_{G}(B) \stackrel{\sim}{\longrightarrow} \operatorname{ker}\left(H^{1}\left(C_{B}, G\right) \rightarrow H^{1}\left(V_{B}^{\prime}, G\right) \times H^{1}\left(\left(\widehat{A \otimes_{R} B}\right)_{I \otimes_{R} B}, G\right)\right) .
$$


5.3. Link with the tangent space. Our goal is to differentiate the mapping $p$ : $L G \rightarrow \operatorname{Bun}_{G}$. Let $B$ be a $R$-algebra and consider the map

$$
p: L G(B[\epsilon]) \rightarrow \operatorname{Bun}_{G}(B[\epsilon]) .
$$

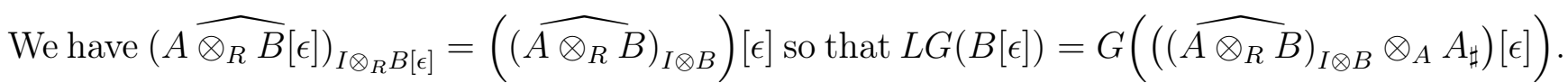

We consider the commutative diagram of categories 1

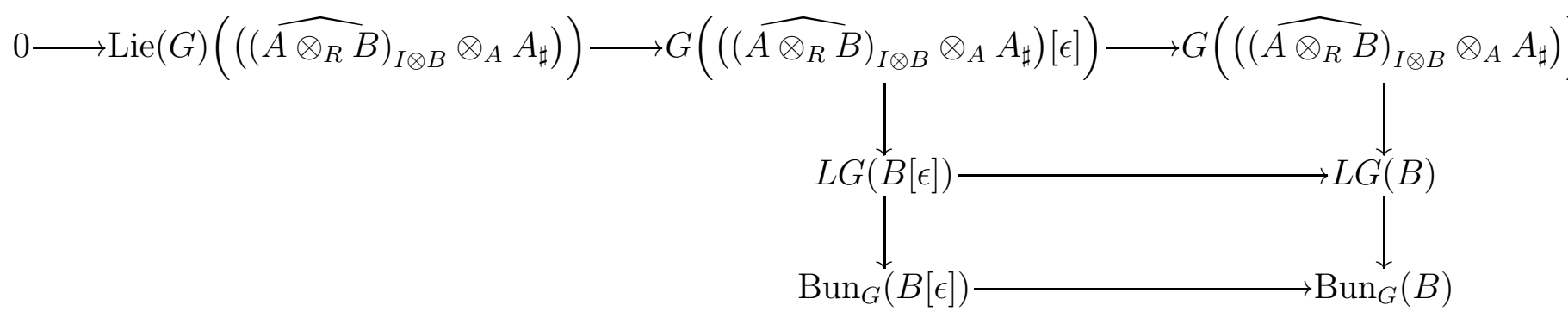

where the first line is the exact sequence defining the Lie algebra. By considering the fiber at the trivial $G$-torsor $b \in \operatorname{Bun}_{G}(B)$, we get then a functor

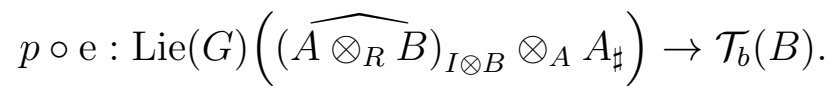

Since $\left.\operatorname{Lie}(G)\left(\left(\widehat{A \otimes_{R} B}\right)_{I \otimes B} \otimes_{A} A_{\sharp}\right)=\mathbf{W}(\mathcal{L} i e(G))\left(\widehat{\left(\widehat{\otimes_{R} B}\right.}\right)_{I \otimes B} \otimes_{A} A_{\sharp}\right)$ we have a $R$-functor

$$
d p: L \mathbf{W}(\mathcal{L} i e(G)) \rightarrow \mathcal{T}_{b}
$$

We use now the equivalence of categories between $\mathcal{T}_{b}(B)$ and $\operatorname{Tors}_{\mathbf{W}(\mathcal{L} i e(G))}\left(C_{B}\right)$ (cf. 4.3) and get the following compatibility with the classifying maps

$$
\begin{aligned}
& L \mathbf{W}(\mathcal{L} i e(G))(B) \longrightarrow \mathbf{W}(\mathcal{L} i e(G))\left(V_{B}^{\prime}\right) \backslash L \mathbf{W}(\mathcal{L} i e(G))(B) / L^{+} \mathbf{W}(\mathcal{L} i e(G))(B) \longrightarrow H^{1}\left(C_{B}, \mathbf{W}(\mathcal{L} i e)(G)\right) \\
& \quad \mathcal{T}_{b}(B) \\
& \text { class map }
\end{aligned}
$$

We observe that the $\mathbf{W}(\mathcal{L} i e(G))$-torsors over affine schemes are trivial so that the top right map is an isomorphism according to the fact $(*)$ above. Also $H^{1}\left(C_{B}, \mathbf{W}(\mathcal{L} i e(G))\right)=$ $H^{1}\left(C_{B}, \mathbf{W}(\mathcal{L} i e(G))\right)$ identifies with the coherent cohomology of the $\mathcal{O}_{S}$-module $\mathcal{L} i e(G)$ [Mn, prop. III.3.7].

5.4. Heinloth's section. This statement is a variation over a local henselian noetherian base of a result due to Heinloth when the residue field is algebraically closed [He1, cor. 8].

\footnotetext{
${ }^{1}$ With the convention that a set defines a groupoid [St, 4.2.7, Tag 001A].
} 
Proposition 5.4. Assume that $S=\operatorname{Spec}(R)$ with $R$ local noetherian henselian with residue field $\kappa$ infinite (resp. finite of characteristic $\neq 2$ ). We assume that $G$ is semisimple and that its fundamental group is smooth over $C$. We assume that $G_{D_{\kappa}}$ admits a strictly proper parabolic $D_{\kappa}$-subgroup (resp. is split).

(1) There exists a map $F: \mathbf{A}_{R}^{n} \rightarrow L G$ such that the composite

$$
f: \mathbf{A}_{R}^{n} \stackrel{F}{\rightarrow} L G \stackrel{p}{\rightarrow} \operatorname{Bun}_{G}
$$

is a map of stacks, maps $0_{R}$ to the trivial $G$-torsor $b$ and such that

$$
d f_{0_{R}}: R^{n} \rightarrow T_{\mathrm{Bun}_{G}, b}(R)
$$

is essentially surjective. Furthermore there exists a neighborhood $\mathcal{N}$ of $0_{\kappa}$ in $\mathbf{A}_{R}^{n}$ such that $f_{\mid \mathcal{N}}$ is smooth.

(2) Let $E$ be a $G$-bundle over $C$ such that $E \times_{C} C_{\kappa}$ is trivial. Then $E$ is trivial on $V^{\prime}$.

Proof. (1) We proceed first to the case of infinite residue field. The proof goes by a differential argument. The $R$-module $H^{1}(C, \operatorname{Lie}(G))$ is finitely generated over $R$ [Ha, III.5.2] and we lift a generating family of $H^{1}(C$, Lie $(G))$ to a family of elements $Y_{1}, \ldots, Y_{r}$ of $\operatorname{Lie}(G)\left(\widehat{A} \otimes_{A} A_{\sharp}\right)$. We have noticed that $\widehat{A}$ is a semilocal noetherian ring (Ex. 5.2.(b)). We want now to apply Lemma 3.2 to $G_{\widehat{A}}$ with respect to the closed points of $\operatorname{Spec}(\widehat{A})$. Since the scheme of parabolic groups of $G$ is smooth [SGA3, XXVI.3.5], the Hensel lemma shows that $G_{D}$ admits a strictly proper parabolic $D$-subgroup scheme. The same argument shows that $G_{\widehat{A}}$ admits a strictly proper parabolic $\widehat{A}$-subgroup scheme $P$. We put $U=\operatorname{rad}(P)$, it is a smooth affine $\widehat{A}$-group scheme. We denote by $U^{\prime}$ its last $R$-subgroup scheme of Demazure's filtration [SGA3, XXVI.2.1]. Lemma 3.2 provides elements $g_{1}, \ldots, g_{m} \in G^{+, P}(\widehat{A})$ such that the product map

$$
h: U^{\prime m} \rightarrow G, \quad\left(u_{1}, \ldots, u_{m}\right) \mapsto{ }^{g_{1}} u_{1} \ldots{ }^{g_{m}} u_{m}
$$

induces a surjective differential

$$
d h: \operatorname{Lie}\left(U^{\prime}\right)^{m}(\widehat{A}) \rightarrow \operatorname{Lie}(G)(\widehat{A}), \quad\left(X_{1}, \ldots, X_{m}\right) \rightarrow{ }^{g_{1}} X_{1}+\cdots+{ }^{g_{m}} X_{m} .
$$

In other words we have

$$
\operatorname{Lie}(G)(\widehat{A})={ }^{g_{1}} \operatorname{Lie}\left(U^{\prime}\right)(\widehat{A})+{ }^{g_{2}} \operatorname{Lie}\left(U^{\prime}\right)(\widehat{A})+\ldots+{ }^{g_{m}} \operatorname{Lie}\left(U^{\prime}\right)(\widehat{A})
$$

so that (using the identity of Lemma 8.3. (2))

$$
\begin{gathered}
\operatorname{Lie}(G)\left(\widehat{A} \otimes_{A} A_{\sharp}\right)=\operatorname{Lie}(G)(\widehat{A}) \otimes_{A} A_{\sharp} \\
={ }^{g_{1}} \operatorname{Lie}\left(U^{\prime}\right)(\widehat{A}) \otimes_{A} A_{\sharp}+{ }^{g_{2}} \operatorname{Lie}\left(U^{\prime}\right)(\widehat{A}) \otimes_{A} A_{\sharp}+\ldots+{ }^{g_{m}} \operatorname{Lie}\left(U^{\prime}\right)(\widehat{A}) \otimes_{A} A_{\sharp} .
\end{gathered}
$$

We can write

$$
Y_{i}=\sum_{j=1, \ldots, m} c_{i, j}{ }^{g_{j}} Z_{i, j}
$$


where $Z_{i, j} \in \operatorname{Lie}\left(U^{\prime}\right)(\widehat{A})$ and $c_{i, j} \in \widehat{A} \otimes_{A} A_{\sharp}$ for each $j$.

Since $U^{\prime}$ is a $\widehat{A}$-vector group scheme, there is a canonical identification exp : $\mathbf{W}\left(\mathcal{L} i e\left(U^{\prime}\right)\right) \stackrel{\sim}{\longrightarrow} U^{\prime}, X \mapsto \exp (X)$. We consider the polynomial ring $B=R\left[t_{i, j}\right]$ where $i=1, \ldots, r, j=1, \ldots, m$. We consider the map of $R$-functors $F: \mathbf{A}_{R}^{r m} \rightarrow L G$ defined by the element

$$
\left.\prod_{i=1, . ., r, j=1, \ldots, m}{ }^{g_{i}} \exp \left(t_{i, j} c_{i, j} Z_{i, j}\right) \in G\left(\widehat{\left(\widehat{\times_{R} B} B\right.}\right)_{I \otimes_{R} B} \otimes_{A} A_{\sharp}\right)=L G(B)
$$

where we can take for example the lexicographic order. It induces a $R$-map $f: \mathbf{A}_{R}^{r m} \rightarrow \operatorname{Bun}_{G}$ of stacks mapping $0_{R}$ to the trivial $G$-bundle. Taking into account the last compatibility of $\$ 5.3$, its differential at $0_{R}$

$$
\text { df }: R^{r m} \rightarrow \mathcal{T}_{b}(R)
$$

factorizes through $L \mathbf{W}(\mathcal{L} i e(G))(B)$. More precisely we have a commutative diagram

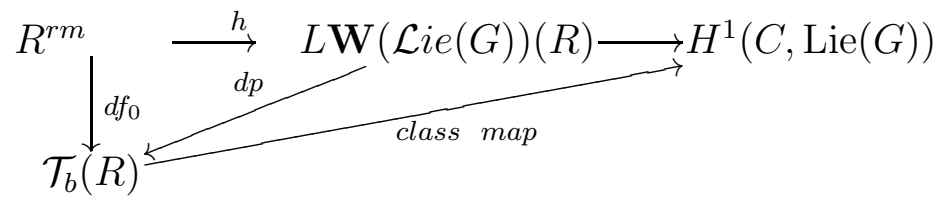

where $h$ maps the basis element $e_{i, j} \in R^{r m}$ to $c_{i, j}{ }^{g_{j}} Z_{i, j}$ in $L \mathbf{W}(\mathcal{L} i e(G))(R)$. We take into account the identity $(* *)$. By $R$-linearity, the image of $R^{m r} \rightarrow H^{1}(C$, Lie $(G))$ contains all $Y_{i}^{\prime}$ 's. Since the $Y_{i}^{\prime}$ 's generate the $R$-module $H^{1}(C, \operatorname{Lie}(G))$, we conclude that $d f_{0}$ is essentially surjective.

The formation of $R^{1} p_{*} \mathcal{L} i e(G)$ commutes with base change, we have an isomorphism $H^{1}(C, \mathcal{L} i e(G)) \otimes_{R} \kappa \stackrel{\sim}{\longrightarrow} H^{1}\left(C_{0}, \mathcal{L} i e(G)\right)$ so that $d f_{0, k}: k^{m r} \rightarrow H^{1}\left(C_{0}, \operatorname{Lie}(G)\right)$ is onto as well.

It follows that $f$ is smooth locally at $0_{\kappa}$ according to the Jacobian smoothness criterion 8.5 stated in the appendix. Thus there is $\mathcal{N}$ as claimed in the statement.

In the finite residue field case, our assumption is stronger so that we can assume that $G_{D}$ is split so that $G_{\widehat{A}}$ admits a strictly Borel $\widehat{A}$-subgroup scheme $B$. Then Lemma 3.2 still work and the proof is verbatim the same.

(2) We see $E$ as an object of $\operatorname{Bun}_{G}(R)$ and consider the fiber product

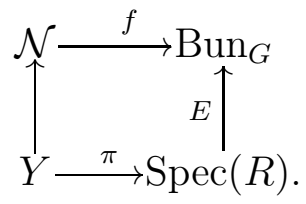

Then $Y$ is an $R$-algebraic space [O, 8.16] which is smooth over $R$. Since Bun $_{G}$ is quasi-separated over $\operatorname{Spec}(R), f$ is quasi-separated (use [St, 92.3, Tag 04XB]). By base change, it follows that $\pi$ is quasi-separated. Since $f\left(0_{\kappa}\right)$ is the trivial bundle which is in the image of $E$ restricted $\kappa, Y(\kappa) \neq \emptyset$. Hence by Hensel Lemma 8.1 for 
algebraic spaces shows that $Y(R) \neq \emptyset$. It follows that there exists $u \in \mathcal{N}(R)$ which maps to $E$. Since the map $\mathcal{N} \rightarrow \operatorname{Bun}_{G}$ factorizes through $L G$, we conclude that the $G$-torsor $E$ is trivial on $V^{\prime}$.

Remark 5.4.1. As pointed out by Gabber, one can use the usual Hensel lemma in the proof of Proposition 5.4. In the proof, let $Q$ be the $G$-torsor over $C_{\mathcal{N}}$ defined by $f: \mathcal{N} \rightarrow \operatorname{Bun}_{G}$. The point is that the algebraic space $Y$ is representable by the $\mathcal{N}$-scheme $\operatorname{Isom}^{b}\left(Q, E_{\mathcal{N}}\right)$ defined in Lemma 4.1 which is affine of finite presentation.

\section{PROOF OF THE MAIN RESUlT}

We need the following Poonen's result [P] as strengthened by Moret-Bailly [M-B2].

Proposition 6.1. Let $F$ be a field. Let $Y$ be an irreducible $F$-scheme of finite type and of positive dimension. We denote by $Y_{0}^{\text {sep }}$ the set of separable closed points of $Y$. Let $X$ be a F-scheme of finite type and let $f: X \rightarrow Y$ be a smooth surjective $F$-morphism. Then the set

$$
\Sigma_{f}(Y)=\left\{y \in Y_{0}^{s e p} \mid X_{y}(F(y)) \neq \emptyset\right\}
$$

is Zariski dense in $Y$.

Proof. Since shrinking is allowed, this reduces to show that the set $\Sigma_{f}(Y)$ is not-empty which is theorem 1.1 of [M-B2].

Lemma 6.2. Let $F$ be field. Let $X$ be an irreducible $F$-scheme of finite type and of dimension $\geq 1$. Let $X_{0}^{\text {sep }}$ be the set of separable closed points of $X$. Let $H$ be a semisimple $X$-group scheme. Then the set

$$
X(s)=\left\{x \in X_{0}^{s e p} \mid H_{F(x)} \text { is split }\right\}
$$

is Zariski dense in $X$.

Proof. We consider the $X$-scheme $Y=\operatorname{Isom}\left(G_{0}, G\right)$ (where $G_{0}$ is the Chevalley form of $G$ ) which is affine smooth over $X$ [SGA3, XXIV.1.9]. Proposition 6.1 applied to $Y \rightarrow X$ yields that $X(s)$ is dense in $X$.

We can proceed to the proof of Theorem 1.1 .

Proof. We assume first that $\kappa$ is infinite or of characteristic $\neq 2$. Let $\Theta$ be set of irreducible components of $C_{\kappa}$ and denote by $C_{\kappa}^{\theta}$ the component attached to $\theta \in \Theta$. Lemma 6.2 provides two fully distinct families of closed separable points $\left(c_{1}^{\theta}\right)_{\theta \in \Theta}$ and $\left(c_{2}^{\theta}\right)_{\theta \in \Theta}$ of $C_{\kappa}$ such that $G_{c_{i}^{\theta}}$ is a split semisimple $\kappa\left(c_{i}^{\theta}\right)$-group for $i=1,2$ and each $\theta \in \Theta$.

(1) Case (I). Since $R$ is henselian, there exists finite étale extensions $R_{i}^{\theta}$ of $R$ which lifts $\kappa\left(c_{i}^{\theta}\right) / \kappa$ and $R_{i}^{\theta}$ is henselian as well [St, 10.148.4, Tag 04GH]. Since $C$ is smooth 
over $R$, Hensel lemma applies to $C \times_{R} R_{i}^{\theta}$ shows that each $c_{i}^{\theta}$ lifts in a closed $R$ subscheme $D_{i}^{\theta} \rightarrow C$ which is finite over $R$. We put $D_{i}=\bigsqcup_{\theta \in \Theta} D_{i}^{\theta}$ for $i=1,2$. Since $C$ is projective over $R$ and $D_{i}$ is semilocal, $D_{i}$ is a closed $R$-subscheme of an affine open $R$-subscheme of $C$. We claim that the scheme $C \backslash D_{i}$ is affine for $i=1,2$.

Since $D_{i, \kappa}$ is an ample divisor of the curve $C_{\kappa}, D_{i}$ is an ample divisor of $C$ and hence $C \backslash D_{i}$ is affine for $i=1,2$ [EGAIII, 4.7.1]. The group $G \times D_{i, \kappa}$ admits a Borel subgroup (resp. is split) for $i=1,2$. Now let $E$ be a $G$-torsor over $C$ such that $E \times{ }_{C} C_{\kappa}$ is trivial. Proposition 5.4. (2) shows that $E_{\mid C \backslash D_{i}}$ is trivial for $i=1$, 2. Since $C=\left(C \backslash D_{1}\right) \cup\left(C \backslash D_{2}\right)$, we conclude that the $G$-torsor $E$ is locally trivial for the Zariski topology.

Case (II). In this case $R$ is a henselian DVR. Lemma 6.2.(1) (resp. 6.2.(2)) provides two fully distinct families of closed separable points $\left(c_{1}^{\theta}\right)_{\theta \in \Theta}$ and $\left(c_{2}^{\theta}\right)_{\theta \in \Theta}$ of $C_{\kappa}$ such that $G_{c_{i}^{\theta}}$ is a split semisimple $\kappa\left(c_{i}^{\theta}\right)$-group for $i=1,2$ and for each $\theta \in \Theta$. According to [L, §8.3, lemma 3.35], there exists an effective Cartier "horizontal" divisor $D_{i}^{\theta}$ of $C$ (note it is finite over $S$ ) such that $C_{\kappa} \cap \operatorname{Supp}\left(D_{i}^{\theta}\right)=c_{i}^{\theta}$ for each $\theta \in \Theta$ and $i=1,2$.

We consider the Cartier effective divisors $D_{i}=\bigsqcup_{\theta \in \Theta} D_{i}^{\theta}$ for $i=1,2, D_{i}$ is finite over $S$. The argument of case (I) shows that $C \backslash D_{i}$ is affine and that $D_{i}$ embeds in an affine $R$-subscheme of $C$. Since $G_{\kappa\left(c_{i}\right)}$ is split, we have that $G \times D_{i, \kappa}$ is split for $i=1,2$ by using the smoothness of the scheme $\operatorname{Isom}\left(G_{0}, G\right)$ (where $G_{0}$ is the Chevalley form of $G$ ). Repeating verbatim the argument of Case (I) finishes the proof.

(2) We apply (1) to the twisted $R$-group scheme ${ }^{E} G$.

It remains to explain the case when $\kappa$ is finite of characteristic 2 . The point is that there is a wide freedom in the points $c_{i}^{\theta}$ which can be chosen of arbitrary large degree over $\mathbb{F}_{2}$ so that Lemma 3.3. (2) holds for the $\kappa\left(c_{i}^{\theta}\right)$ and Lemma 3.2 holds as well. By inspection of the proof, it follows that Theorem 1.1 holds also in this case.

Remark 6.3. In the proof of (1), an important step is the construction of the divisor $D$ such that $D$ is finite étale over $R$ and $G_{D}$ is split. Though our contruction is quite different, a similar argument has been used by Panin and Fedorov in their proof of Grothendieck-Serre's conjecture [F-P, prop. 4.1].

We have the following refinement of Theorem 1.1 which answers a question of Olivier Benoist.

Theorem 6.4. Assume that $R$ is local henselian noetherian of residue field $\kappa$. Let $f: C \rightarrow \operatorname{Spec}(R)$ be a smooth projective curve such that $C$ is integral and has geometrically connected fibers. Let $G$ be a semisimple simply connected $C$-group scheme. Let $E$ be a $G$-torsor over $C$. Then the following are equivalent:

(i) The $G$-torsor $E$ is locally trivial for the Zariski topology;

(ii) The $G_{C_{\kappa}}$-torsor $E_{C_{\kappa}}$ is locally trivial for the Zariski topology. 
Proof. The implication $(i) \Longrightarrow(i i)$ is obvious. Conversely we assume that $E_{C_{\kappa}}$ is locally trivial for the Zariski topology. Then there exists $V, D$, etc.. as in $\$ 5$ such that the $G$-torsors $E_{V_{\kappa}^{\prime}}$ and $E_{D_{\kappa}}$ are trivial.

Claim 6.5. The $G$-torsor $E_{C_{\kappa}}$ extends to a $G$-torsor $F$ whose restrictions to $V^{\prime}$ and $D$ are trivial.

We postpone the proof of the Claim. Assuming the Claim, the $G$-torsors $E$ and $F$ are isomorphic on $C_{\kappa}$. Theorem 1.1.(2) shows that $E$ and $F$ are locally isomorphic for the Zariski topology. It follows that $E_{V^{\prime}}$ is locally trivial. By varying the choices of $D$, we can find a cover of $C$ by open subsets $V_{1}^{\prime}, \ldots, V_{r}^{\prime}$ such that $E_{V_{i}^{\prime}}$ is locally trivial for $i=1, \ldots, r$. Thus $E$ is locally trivial for the Zariski topology.

For proving the Claim, we use the uniformization bijections

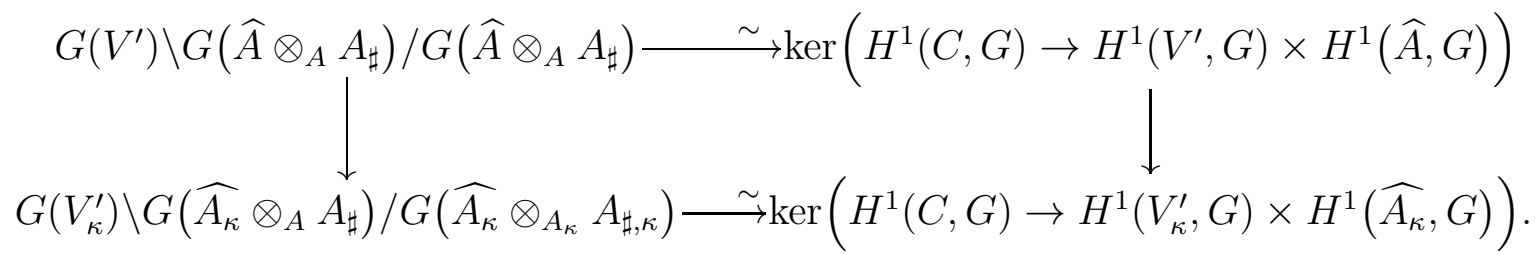

using the notations of $\$ 5$. The $G_{C_{\kappa}}$-torsor $E_{\kappa}$ arises then from an element $g \in$ $G\left(\widehat{A_{\kappa}} \otimes_{A_{\kappa}} A_{\sharp, \kappa}\right)$. Under our assumptions the ring $\widehat{A_{\kappa}}$ is a product of complete DVR's $A_{1} \times \cdots \times A_{t}$ so that $\widehat{A_{\kappa}} \otimes_{A} A_{\sharp}=F_{1} \times \cdots \times F_{t}$ where $F_{i}$ is the fraction field of $A_{i}$. We denote by $\kappa_{i}$ the residue field of $A_{i}$ for $i=1, \ldots, t_{0}, \ldots t$ and may assume that $G_{\kappa_{i}}$ is anisotropic for $i=1, \ldots, t_{0}$ and isotropic for $i=t_{0}+1, \ldots, t$.

We write $g=\left(g_{1}, \ldots, g_{t}\right)$ with $g_{i} \in G\left(F_{i}\right)$. According to [Gi2, Lemme 4.5.(1)], we have $G\left(F_{i}\right)=G\left(F_{i}\right)^{+} G\left(A_{i}\right)$ for $i=1, \ldots, t$. We can then assume that $g_{i}$ belongs to $G\left(F_{i}\right)^{+} i=1, \ldots, t$ (note that $g_{i}=1$ if $G_{F_{i}}$ is anisotropic). Let $P_{i}$ be a minimal $\kappa_{i}$-parabolic subgroup of $G_{\kappa_{i}}$. This lifts in a $\widehat{A_{\kappa}}$-parabolic subgroup of $P$ of $G_{D}$. Since the map $\widehat{A} \rightarrow \widehat{A_{\kappa}}$ is onto (by the Mittag-Leffler's condition), it follows that the map $\widehat{A} \otimes_{A} A_{\sharp} \rightarrow \widehat{A_{\kappa}} \otimes_{A_{\kappa}} A_{\sharp, \kappa}$ is onto. The same argument as in Claim 3.4 shows that the map

$$
G_{D}^{P,+}\left(\widehat{A} \otimes_{A} A_{\sharp}\right) \rightarrow G_{D_{\kappa},+}^{P_{\kappa}}\left(\widehat{A_{\kappa}} \otimes_{A_{\kappa}} A_{\sharp, \kappa}\right)=\prod_{i=1}^{t} G^{+}\left(F_{i}\right)
$$

is onto. Thus $g=\left(g_{i}\right)$ lifts to an element of $G\left(\widehat{A} \otimes_{A} A_{\sharp}\right)$ and the diagram above of uniformization maps yields the Claim.

Remarks 6.6. (a) In case (II) with $G$ semisimple simply connected, the same argument will work provided $D_{\kappa}$ is contained in the smooth locus of $C_{\kappa}$.

(b) In the case $k$ is algebraically closed, it is well-known that $G$-torsors over $C_{\kappa}$ are locally trivial for the Zariski topology. Theorem 6.4 provides then an alternative proof of Drinfeld-Simpson's theorem in this case. 
Corollary 6.7. Assume that $R$ is a DVR with finite residue field $\kappa$. Let $f: C \rightarrow$ $\operatorname{Spec}(R)$ be a smooth projective curve such that $C$ is integral and has geometrically connected fibers. Let $G$ be a semisimple simply connected $C$-group scheme. Then $H_{Z a r}^{1}(C, G)=H^{1}(C, G)$.

Proof. This follows from the fact that $H_{Z a r}^{1}\left(C_{\kappa}, G\right)=H^{1}\left(C_{\kappa}, G\right)$. According to Harder [Hd2], we have $H^{1}\left(\kappa\left(C_{\kappa}\right), G\right)=1$. Nisnevich's theorem [N] (see also [Gu]) shows that $H_{Z a r}^{1}\left(C_{\kappa}, G\right)=H^{1}\left(C_{\kappa}, G\right)$.

Corollary 6.8. Assume that $R$ is a DVR with residue field $\kappa$. Let $f: C \rightarrow \operatorname{Spec}(R)$ be a smooth projective curve such that $C$ is integral and has geometrically connected fibers. Let $G$ be a semisimple simply connected $C$-group scheme. Let $F$ be the function field of $C$ and $G$ a semisimple simply connected $C$-group scheme. Then, the local-global principle holds for $G$-torsors over $F$ with respect to all discrete valuations arising from codimension one points of $C$.

Proof. Let $\xi \in H^{1}(F, G)$ which is trivial over $F_{v}$ for all completions at discrete valuations arising from codimension one points of $C$. By glueing [GP, cor. A.8], there is an element $\zeta \in H^{1}(U, G)$ which maps to $\xi$ over $F$ where $U \subset C$ contains all points of codimension 1. According to [C-T-S1, th. 6. 13], we have $H^{1}(C, G)=H^{1}(U, G)$ so that we an assume that $U=X$. Since $\xi$ is trivial over the completion $F_{w}$ of $F$ at the discrete valuation $w$ associated to the special fiber of $C$, we claim that the specialisation $\zeta_{\kappa} \in H^{1}\left(C_{\kappa}, G\right)$ of $\zeta$ is generically trivial. This follows of the fact that $H^{1}\left(O_{w}, G\right)$ injects in $H^{1}\left(F_{w}, G\right)$ due to Bruhat-Tits (see [Gi1, I.1.2.2]). According to Nisnevich's theorem [N] (see also $\mathrm{Gu}]$ ) this class is Zariski locally trivial on $C_{\kappa}$. Theorem 6.4 enables us to conclude that $\eta$ is Zariski locally trivial and hence $\xi$ is trivial.

Remarks 6.9. (a) The only case where we knew that local-global principle for $G$ simply connected group defined over $C$ (for arbitrary residue fields) is when $G_{F}$ is $F$-rational. In this case, Harbater, Hartmann and Krashen established their "patching local-global principle" [H-H-K, th. 3.7] which implies our local-global principle according to [C-T-P-S, Th. 4.2.(ii)].

(b) The special case when $R$ is the ring of integers of a $p$-adic field was already known [C-T-P-S, Th. 4.8].

The case of a smooth curve defined over a field is of special interest.

Corollary 6.10. Let $X$ be a geometrically connected smooth projective algebraic curve defined over a field $k$. Let $R$ be a local noetherian henselian $k$-ring and $\kappa$ its residue field. Let $G$ be a semisimple simply connected $R$-group scheme.

(1) Let $E$ be a $G$-torsor over $X_{R}$ such that $E \times_{X_{R}} X_{\kappa}$ is locally trivial for the Zariski topology. Then $E$ is locally trivial for the Zariski topology. 
(2) Let $E, E^{\prime}$ be $G$-torsor over $X_{R}$ such that $E \times_{X_{R}} X_{\kappa}$ is isomorphic to $E \times_{X_{R}} X_{\kappa}$ locally for the Zariski topology. Then $E$ and $E^{\prime}$ are locally isomorphic for the Zariski topology.

\section{EXTENSION TO REDUCTIVE GROUPS}

We gather here our results in a single long statement.

Theorem 7.1. Assume that $S=\operatorname{Spec}(R)$ with $R$ local henselian noetherian of residue field $\kappa$. Let $p$ be the characteristic exponent of $\kappa$. Let $f: C \rightarrow \operatorname{Spec}(R)=S$ be a flat projective curve which is integral. Assume that one of the following holds:

(I) $C$ is smooth with geometrically connected fibers;

(II) $R$ is a DVR and $\mathcal{O}_{S} \rightarrow f_{*} \mathcal{O}_{X}$ is universally an isomorphism.

Let $G$ be a reductive $C$-group scheme and consider its presentation [SGA3, XXII.6.2.3]

$$
1 \rightarrow \mu \rightarrow G^{s c} \times_{C} \operatorname{rad}(G) \rightarrow G \rightarrow 1
$$

where $\operatorname{rad}(G)$ is the radical $C$-torus of $G$ and $G^{s c}$ is the simply connected universal cover of $D G$. We assume that

(i) $\mu$ is étale over $C$;

(ii) the $C$-torus $\operatorname{rad}(G)$ is split by a $p^{\prime}$-Galois cover of the shape

$$
X \stackrel{\pi}{\rightarrow} C \times{ }_{S} \operatorname{Spec}\left(R^{\prime}\right) \rightarrow C
$$

where $R^{\prime} / R$ is a connected Galois cover and $\pi$ is a finite Galois cover such that the map $\mathcal{O}_{S^{\prime}} \rightarrow g_{*} \mathcal{O}_{X}$ is universally an isomorphism where $g=p_{2} \circ \pi: X \rightarrow S^{\prime}=\operatorname{Spec}\left(R^{\prime}\right)$.

Then the following hold:

(1) Let $E$ be a $G$-torsor over $C$ such that $E \times{ }_{C} C_{\kappa}$ is trivial. Then $E$ is locally trivial for the Zariski topology.

(2) Let $E, E^{\prime}$ be two $G$-torsors over $C$ such that $E \times{ }_{C} C_{\kappa}$ is isomorphic to $E^{\prime} \times_{C} C_{\kappa}$. Then $E$ and $E^{\prime}$ are locally isomorphic for the Zariski topology.

Proof. We consider the following commutative diagram

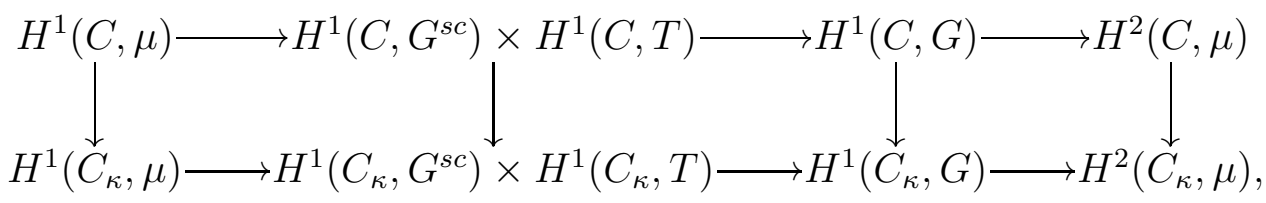

where the horizontal lines are exact sequences of pointed sets. On the other hand, the proper base change theorem for étale cohomology shows that the maps $H^{i}(C, \mu) \rightarrow$ $H^{i}\left(C_{\kappa}, \mu\right)$ are bijective for $i=1,2$ [SGA4, XII.5.5.(iii)]. By diagram chase, it follows that the map

$$
\begin{gathered}
\operatorname{ker}\left(H^{1}\left(C, G^{s c}\right) \rightarrow H^{1}\left(C_{\kappa}, G^{s c}\right)\right) \times \operatorname{ker}\left(H^{1}(C, T) \rightarrow H^{1}\left(C_{\kappa}, T_{\kappa}\right)\right) \\
\rightarrow \operatorname{ker}\left(H^{1}(C, G) \rightarrow H^{1}\left(C_{\kappa}, G\right)\right)
\end{gathered}
$$


is onto. The first kernel (resp. the second one) consists of Zariski locally trivial according to Theorem 1.1 (resp. Proposition 2.2.(3) and Corollary 2.3). Thus the third kernel consists of Zariski locally trivial. The assertion (2) follows by twisting.

\section{Appendix: FaCts on SMOothness}

The purpose of this appendix is to provide proofs to statements for algebraic spaces which are well-known among experts.

8.1. Hensel lemma for algebraic spaces. Rydh proved several generalizations for étale morphisms of algebraic spaces from the case of schemes [Ry, app. A] including the Hensel lemma. Our purpose here is to prove the following variant of [EGAIV, 18.5.17].

Proposition 8.1. Let $R$ be a henselian local ring with residue field $\kappa$. Let $f: X \rightarrow Y$ be a smooth morphism of (quasi-separated) $R$-algebraic spaces. Let $y \in Y(R)$ and let $x_{0} \in X(\kappa)$ with the image $y_{0}$ of $y$ by $Y(R) \rightarrow Y(\kappa)$ is the image of $x_{0}$ by $X(\kappa) \rightarrow Y(\kappa)$. Then there exists $x \in X(R)$ mapping to $y$ and $x_{0}$.

Proof. Up to pull-back everything by $y: \operatorname{Spec}(R) \rightarrow Y$, we can assume that $Y=$ $\operatorname{Spec}(R)$. Now we use that there is an étale morphism $f: U \rightarrow X$ where $U$ is an affine scheme such that $x_{0}=f\left(u_{0}\right)$ for some $u_{0} \in U(\kappa)$ [K, II.6.4]. By composition, the morphism $U \rightarrow Y=\operatorname{Spec}(R)$ is smooth and the usual Hensel lemma applies [EGAIV, 18.5.17].

8.2. Lie algebra of a $S$-group space. Let $f: X \rightarrow Y$ be a morphism of $S$-algebraic spaces. We consider the quasi-coherent sheaf $\Omega_{X / Y}^{1}$ on $X$ defined in [St, 68.1.2, Tag 04CT]. Let $T$ be a $S$-scheme equipped with a closed subscheme $T_{0}$ defined by a quasi-coherent ideal $\mathcal{I}$ such that $\mathcal{I}^{2}=0$. According to [O, 7.A page 167] for any commutative diagram of algebraic spaces

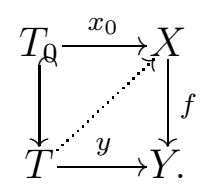

if there exists a dotted arrow filling in the diagram then the set of such dotted arrows form a torsor under $\operatorname{Hom}_{\mathcal{O}_{T_{0}}}\left(x_{0}^{*} \Omega_{X / Y}^{1}, \mathcal{I}\right)$. We extend to group spaces well-known statements on group schemes [SGA3, II.4.11.3].

Lemma 8.2. Let $G$ be a $S$-group space. We denote by $e_{G}: S \rightarrow G$ the unit point and put $\omega_{G / S}=e_{G}^{*}\left(\Omega_{G / S}^{1}\right)$.

(1) There is a canonical isomorphism of $S$-functors $\operatorname{Lie}(G) \stackrel{\sim}{\longrightarrow} \mathbf{V}\left(\omega_{G / S}\right)$ which is compatible with the $\mathcal{O}_{S}$-structure. 
(2) If $\omega_{G / S}$ is a locally free coherent sheaf, then $\operatorname{Lie}(G) \stackrel{\sim}{\longrightarrow} \mathbf{W}\left(\omega_{G / S}^{\vee}\right)$. In particular we have an isomorphism

$$
\operatorname{Lie}(G)(R) \otimes_{R} R^{\prime} \stackrel{\sim}{\longrightarrow} \operatorname{Lie}(G)\left(R^{\prime}\right)
$$

for each morphism of $S$-rings $R \rightarrow R^{\prime}$.

(3) Assume that $G$ is smooth and quasi-separated over $S$. Then $\omega_{G / S}$ is a finite locally free coherent sheaf and (2) holds.

Under the conditions of (2) or (3), we denote also by $\mathcal{L} i e(G)=\omega_{G / S}^{\vee}$ the locally free coherent sheaf.

Proof. (1) Let $T_{0}$ be a $S$-scheme and consider $T=T_{0}[\epsilon]$. We apply the above fact to the morphism $G \rightarrow S$ and the points $x_{0}=e_{G_{T_{0}}}$ and $y: T \rightarrow S$ the structural morphism. It follows that $\operatorname{ker}\left(G(T) \rightarrow G\left(T_{0}\right)\right)$ is a torsor under $\operatorname{Hom}_{\mathcal{O}_{T_{0}}}\left(e_{G_{T_{0}}}^{*} \Omega_{G / S}^{1}, \epsilon \mathcal{O}_{T_{0}}\right) \cong$ $\operatorname{Hom}_{\mathcal{O}_{T_{0}}}\left(e_{G_{T_{0}}}^{*} \Omega_{G / S}^{1}, \mathcal{O}_{T_{0}}\right)=\operatorname{Hom}_{\mathcal{O}_{T_{0}}}\left(\omega_{G / S}^{1} \otimes_{\mathcal{O}_{S}} \mathcal{O}_{T_{0}}, \mathcal{O}_{T_{0}}\right)$. We have constructed a isomorphism of $S$-functors $\operatorname{Lie}(G) \stackrel{\sim}{\longrightarrow} \mathbf{V}\left(\omega_{G / S}\right)$ and the compatibility of $\mathcal{O}_{S}$-structures is a straightforward checking.

(2) If $\omega_{G / S}$ is a locally free coherent sheaf, then $\operatorname{Lie}(G) \stackrel{\sim}{\longrightarrow} \mathbf{V}\left(\omega_{G / S}\right) \stackrel{\sim}{\longrightarrow} \mathbf{W}\left(\omega_{G / S}^{\vee}\right)$. The next fact follows from [EGAIII, 12.2.3].

(3) According to [St, 68.7.16, Tag 0CK5], $\Omega_{G / S}^{1}$ is a finite locally free coherent sheaf over $G$. If follows that $\omega_{G / S}^{1}$ is a finite locally free coherent sheaf over $S$.

Lemma 8.3. Let $G$ be a smooth $S$-group space and let $T$ be a $S$-scheme equipped with a closed subscheme $T_{0}$ defined by a quasi-coherent ideal $\mathcal{I}$ such that $\mathcal{I}^{2}=0 . W e$ denote by $t_{0}: T_{0} \rightarrow S$ the structural morphism, $G_{0}=G \times_{S} T_{0}$ and assume that $t_{0}$ is quasi-compact and quasi-separated.

(1) We have an exact sequence of fppf (resp. étale, Zariski) sheaves on $S$

$$
0 \rightarrow \mathbf{W}\left(\left(t_{0}\right)_{*}\left(\mathcal{L} i e\left(G_{0}\right) \otimes_{\mathcal{O}_{T_{0}}} \mathcal{I}\right)\right) \rightarrow \prod_{T / S} G \rightarrow \prod_{T_{0} / S} G \rightarrow 1
$$

(2) If $T=\operatorname{Spec}(A)$ is affine and $T_{0}=\operatorname{Spec}(A / I)$, we have an exact sequence

$$
0 \rightarrow \operatorname{Lie}(G)(A) \otimes_{A} I \rightarrow G(A) \rightarrow G(A / I) \rightarrow 1 .
$$

Proof. (1) We have

$$
\operatorname{Hom}_{\mathcal{O}_{T_{0}}}\left(\omega_{G_{0} / T_{0}}, \mathcal{I}\right)=H^{0}\left(T_{0}, \mathcal{L} i e\left(G_{0}\right) \otimes_{\mathcal{O}_{T_{0}}} \mathcal{I}\right)=H^{0}\left(T, t_{0, *}\left(\mathcal{L} i e\left(G_{0}\right) \otimes_{\mathcal{O}_{T_{0}}} \mathcal{I}\right)\right),
$$

whence an exact sequence

$$
0 \rightarrow H^{0}\left(T, t_{0, *}\left(\mathcal{L} i e\left(G_{0}\right) \otimes_{\mathcal{O}_{T_{0}}} \mathcal{I}\right)\right) \rightarrow G(T) \rightarrow G\left(T_{0}\right)
$$


Now let $h: S^{\prime} \rightarrow S$ be a flat morphism locally of finite presentation and denote by $G^{\prime}=G \times{ }_{S} G^{\prime}, h_{T}: T^{\prime} \rightarrow T, \ldots$ the relevant base change to $S^{\prime}$. Since $t_{0}$ is quasicompact and quasi-separated, the flatness of $h$ yields an isomorphism [St, 28.5.2, Tag $02 \mathrm{KH}]$

$$
h^{*}\left(t_{0, *}\left(\mathcal{L} i e\left(G_{0}\right) \otimes_{\mathcal{O}_{T_{0}}} \mathcal{I}\right)\right) \stackrel{\sim}{\longrightarrow} t_{0, *}^{\prime}\left(h_{T_{0}}^{*}\left(\mathcal{L} i e\left(G_{0}\right) \otimes_{\mathcal{O}_{T_{0}}} \mathcal{I}\right)\right)=t_{0, *}^{\prime}\left(\mathcal{L} i e\left(G_{0}^{\prime}\right) \otimes_{\mathcal{O}_{T_{0}^{\prime}}} \mathcal{I}^{\prime}\right) .
$$

The similar sequence for $T_{0}^{\prime}$ reads then

$$
0 \rightarrow H^{0}\left(T^{\prime}, t_{0, *}\left(\mathcal{L} i e\left(G_{0}\right) \otimes_{\mathcal{O}_{T_{0}}} \mathcal{I}\right)\right) \rightarrow G\left(T^{\prime}\right) \rightarrow G\left(T_{0}^{\prime}\right) .
$$

We have then an exact sequence of fppf sheaves

$$
0 \rightarrow \mathbf{W}\left(\left(t_{0}\right)_{*}\left(\mathcal{L} i e\left(G_{0}\right) \otimes_{\mathcal{O}_{T_{0}}} \mathcal{I}\right)\right) \rightarrow \prod_{T / S} G \rightarrow \prod_{T_{0} / S} G
$$

For $T$ an affine scheme, the map $G(T) \rightarrow G\left(T_{0}\right)$ is onto since the smooth $S$-group space $G$ is formally smooth [St, 68.19.6, Tag 04AM]. It implies the exactness for the the Zariski, étale and fppf topologies.

(2) We can assume that $S=T=\operatorname{Spec}(A)$. In this case, we have

$$
H^{0}\left(S,\left(t_{0}\right)_{*}\left(\mathcal{L} i e\left(G_{0}\right) \otimes_{\mathcal{O}_{T_{0}}} \mathcal{I}\right)\right)=H^{0}\left(T_{0}, \mathcal{L} i e\left(G_{0}\right) \otimes_{\mathcal{O}_{T_{0}}} \mathcal{I}\right)=\operatorname{Lie}\left(G_{0}\right)(A / I) \otimes_{A / I} I .
$$

We have $\operatorname{Lie}\left(G_{0}\right)(A / I)=\operatorname{Lie}(G)(A) \otimes_{A} A / I$ in view of Lemma 8.2. (2) whence the identification $\operatorname{Lie}\left(G_{0}\right)(A / I) \otimes_{A / I} I \cong \operatorname{Lie}(G)(A) \otimes_{A} I$. Then (1) provides the exact sequence

$$
0 \rightarrow \operatorname{Lie}(G)(A) \otimes_{A} I \rightarrow G(A) \rightarrow G(A / I)
$$

and the right map is onto since $G$ is smooth.

Remarks 8.4. (a) A special case of (1) is $T=S[\epsilon]$ and $T_{0}=S$. We get an exact sequence of fppf (resp. étale, Zariski) sheaves on $S$

$$
0 \rightarrow \mathbf{W}(\mathcal{L} i e(G)) \rightarrow \prod_{S[\epsilon] / S} G \rightarrow G \rightarrow 1
$$

(b) In the group scheme case, (2) is established in [D-G, proof of II.5.2.8].

8.3. Jacobian criterion for stacks. Let $S$ be a scheme and let $\mathcal{X}, \mathcal{Y}$ be quasiseparated algebraic $S$-stacks of finite presentation. Let $g: \mathcal{X} \rightarrow \mathcal{Y}$ be a 1-morphism over $S$. We have a 1 -morphism $T g: T(\mathcal{X}) \rightarrow T(\mathcal{Y})$ of algebraic stacks [L-M-B, 17.14, 17.16].

Let $s \in S$ and denote by $K$ the residue field of $s$. Let $x: \operatorname{Spec}(K) \rightarrow \mathcal{X}$ be a 1-morphism mapping to $s$. We put $T(\mathcal{X})_{x}=T(\mathcal{X} / S) \times_{\mathcal{X}} \operatorname{Spec}(K)$ and denote by $\operatorname{Tan}_{x}(\mathcal{X})$ the category $T(\mathcal{X})_{x}(K)$. We denote by $y=h \circ x: \operatorname{Spec}(K) \rightarrow \mathcal{Y}$ and get the tangent morphism $(T g)_{x}: \operatorname{Tan}_{x}(\mathcal{X}) \rightarrow \operatorname{Tan}_{y}(\mathcal{Y})$. 
Proposition 8.5. We assume that $\mathcal{X}$ is smooth at $x$ over $S$. Then the following assertions are equivalent:

(i) The morphism $g$ is smooth at $x$;

(ii) The tangent morphism $(\mathrm{Tg})_{x}: \operatorname{Tan}_{x}(\mathcal{X}) \rightarrow \operatorname{Tan}_{y}(\mathcal{Y})$ is essentially surjective.

Furthermore, under those conditions, $\mathcal{Y}$ is smooth at $y$ over $S$.

Proof. In the case of a morphism $g: X \rightarrow Y$ of $S$-schemes locally of finite presentation such that $g(x)=y$ and $X$ is smooth at $x$ over $S$, we have that $K=\kappa(x)=\kappa(y)$ so that the statement is a special case of [EGAIV, 17.11.1]. We proceed now to the stack case.

$(i) \Longrightarrow($ ii $)$. Up to shrinking, we can assume that $\mathcal{X}$ is smooth over $S$ and that $g$ is smooth. Also $g$ is formally smooth [St, 98.8.4, Tag 0DNV] that is satisfies the relevant infinitesimal lifting criterion. It applies in particular to $K[\epsilon]$ whence the essential surjectivity of the tangent morphism.

$(i i) \Longrightarrow(i)$. According to [L-M-B, Thm. 6.3], there exists a smooth 1 -morphism $\varphi: Y \rightarrow \mathcal{Y}$ and a point $y_{1} \in Y(K)$ mapping to $y$ such that $Y$ is an affine scheme. We note that $K=\kappa\left(y_{1}\right)$. We consider the fiber product $\mathcal{X}^{\prime}=\mathcal{X} \times \mathcal{Y} Y$, it is an algebraic stack and there exists a 1 -morphism $x^{\prime}: \operatorname{Spec}(K) \rightarrow \mathcal{X}^{\prime}$ lifting $x$ and $y_{1}$. There exists a smooth 1-morphism $\psi: X^{\prime} \rightarrow \mathcal{X}^{\prime}$ and a point $x_{1} \in X^{\prime}(K)$ mapping to $x$ such that $X^{\prime}$ is an affine scheme. By construction we have again that $K=\kappa\left(x_{1}\right)$. We have then the commutative diagram

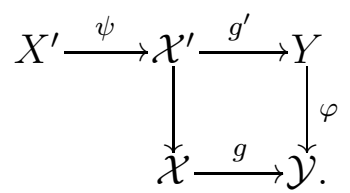

According to [L-M-B, Lem. 17.5.1], the square

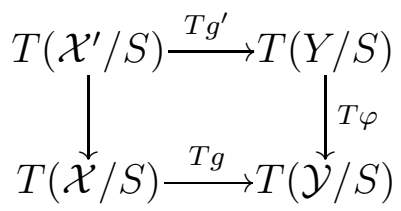

is $2-$ cartesian. It follows that the square

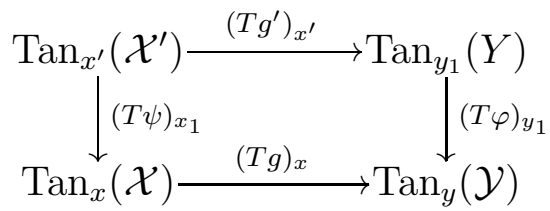

is 2-cartesian. Our assumption is that the bottom morphism is essentially surjective, it follows that $\left(T g^{\prime}\right)_{x^{\prime}}: \operatorname{Tan}_{x_{1}}\left(\mathcal{X}^{\prime}\right) \rightarrow \operatorname{Tan}_{y_{1}}(Y)$ is essentially surjective as well. Since $\psi$ is smooth, the map $(T \psi)_{x_{1}}: \operatorname{Tan}_{x^{\prime}}\left(X^{\prime}\right) \rightarrow \operatorname{Tan}_{x_{1}}\left(\mathcal{X}^{\prime}\right)$ is essentially surjective. By 
composition it follows that $\operatorname{Tan}_{x_{1}}\left(X^{\prime}\right) \rightarrow \operatorname{Tan}_{y_{1}}(Y)$ is essentially surjective. Since $X^{\prime}$ and $Y$ are locally of finite presentation over $S$, the case of schemes yields that $g^{\prime} \circ \psi: X^{\prime} \rightarrow Y$ is smooth at $x^{\prime}$. By definition of smoothness for morphisms of stacks [O, §8.2], we conclude that $g$ is smooth at $x$.

We assume (ii) and shall show that $\mathcal{Y}$ is smooth at $y$ over $S$. Using the diagrams of the proof, we have seen that the $S$-morphism $X^{\prime} \rightarrow Y$ of schemes is smooth at $x^{\prime}$. Once again the classical Jacobian criterion [EGAIV, 17.11.1] applies and shows that $Y$ is smooth at $y_{1}$ over $S$. By definition of smoothness for stacks, we get that $\mathcal{Y}$ is smooth at $y$ over $S$.

\section{REFERENCES}

[B-L] A. Beauville, Y. Laszlo, Un lemme de descente, C. R. Acad. Sci. Paris Sér. I Math., 320 (1995), 335-340.

[B-F1] P. Belkale, N. Fakhruddin, Triviality properties of principal bundles on singular curves, Algebr. Geom. 6 (2019), 234-259.

[B-F2] P. Belkale, N. Fakhruddin, Triviality properties of principal bundles on singular curves-II, preprint, arXiv:1801.04784.

[Bh] B. Bhatt, Algebraization and Tannaka duality, Camb. J. Math. 4 (2016), 403-461.

[B-T] A. Borel, J. Tits, Homomorphismes "abstraits" de groupes algébriques simples, Ann. of Math. 97 (1973), 499-571.

[B:AC] N. Bourbaki, Algèbre commutative, Ch. 1 à 10, Springer.

[C-S-U-W] A.M. Cohen, A. Steinbach, R. Ushirobira, D. Wales, Lie algebras generated by extremal elements, J. Algebra 236 (2001), 122-154.

[C-T-P-S] J.-L. Colliot-Thélène, R. Parimala, V. Suresh, Patching and local-global principles for homogeneous spaces over function fields of p-adic curves, Comment. Math. Helv. 87 (2012), 1011-1033.

[C-T-S1] J.-L. Colliot-Thélène, J.-J. Sansuc, Fibrés quadratiques et composantes connexes réelles, Mathematische Annalen 244 (1979), 105-134.

[C-T-S2] J.-L. Colliot-Thélène, J.-J. Sansuc, Principal Homogeneous Spaces under Flasque Tori: Applications, Journal of Algebra 106 (1987), 148-205.

[C-G-P] B. Conrad, O. Gabber, G. Prasad, Pseudo-reductive groups, Cambridge University Press, second edition (2016).

[D-G] M. Demazure, P. Gabriel, Groupes algébriques, North-Holland (1970).

[D-S] V. G. Drinfeld, C. Simpson, B-structures on G-bundles and local triviality, Mathematical Research Letters 2 (1995), 823-829.

[EGAI] A. Grothendieck, J.-A. Dieudonné, Eléments de géométrie algébrique. I, Grundlehren der Mathematischen Wissenschaften 166; Springer-Verlag, Berlin, 1971.

[EGAII] A. Grothendieck (avec la collaboration de J. Dieudonné), Eléments de Géométrie Algébrique II, Publications mathématiques de l'I.H.É.S. no 8 (1961).

[EGAIII] A. Grothendieck (avec la collaboration de J. Dieudonné), Eléments de Géométrie Algébrique II, Publications mathématiques de l'I.H.É.S. no 11 (1961) and 17 (1963).

[EGAIV] A. Grothendieck (avec la collaboration de J. Dieudonné), Eléments de Géométrie Algébrique IV, Publications mathématiques de l'I.H.É.S. no 20, 24, 28 and 32 (1964 - 1967). 
[F-P] R. Fedorov, I. Panin, A proof of the Grothendieck-Serre conjecture on principal bundles over regular local rings containing infinite fields, Publications Mathématiques de l'I.H.É.S 122 (2015), 169-193.

[F-R] D. Ferrand and M. Raynaud, Fibres formelles d'un anneau local noethérien, Ann. Sci. ÃL'cole Norm. Sup. 3 (1970), 295-311.

[FGA] A. Grothendieck, Fondements de la géométrie algébrique, (Extraits du Séminaire Bourbaki, 1957-1962), Paris, Secrétariat mathématique, 1962.

[Gi1] P. Gille, R-équivalence et torseurs sur la droite affine, Orsay (1994).

[Gi2] P. Gille, Le problème de Kneser-Tits, exposé Bourbaki n0 983, Astérisque 326 (2009), 39-81.

[GP] P. Gille, A. Pianzola, Isotriviality and étale cohomology of Laurent polynomial rings, Journal of Pure and Applied Algebra 212 (2008), 780-800.

[Gd] J. Giraud, Cohomologie non-abélienne, Springer (1970).

[Gu] N. Guo, The Grothendieck-Serre Conjecture over Semilocal Dedekind Rings, preprint arXiv:1902.02315.

[H-H-K] D. Harbater, J. Hartmann, D. Krashen, Applications of patching to quadratic forms and central simple algebras, Invent. Math. 178 (2009), 231-263.

[Hd1] G. Harder, Halbeinfache Gruppenschemata über vollständigen Kurven, Inventiones mathematicae 6 (1968), 107-149.

[Hd2] G. Harder, Über die Galoiskohomologie halbeinfacher algebraischer Gruppen. III, J. reine angew. math. 274-275 (1975), 125-138.

[Ha] R. Hartshorne, Algebraic Geometry, Graduate Texts in Mathematics, Springer.

[He1] J. Heinloth, Uniformization of G-bundles, Math. Annalen 347 (2010), 499-528.

[He2] J. Heinloth, Lectures on the moduli stack of vector bundles on a curve, Affine flag manifolds and principal bundles, 123-153, Trends Math., Birkhäuser/Springer Basel AG, Basel, 2010.

[I] L. Illusie, Grothendieck's existence theorem in formal geometry, with a letter of Jean-Pierre Serre. Math. Surveys Monogr., 123, Fundamental algebraic geometry, 179-233, Amer. Math. Soc., Providence, RI, 2005.

[Ks] M. Knus, Quadratic and Hermitian Forms over Rings, Grundlehren der mathematischen Wissenschaften 294 (1991), Springer.

[K] D. Knutson, Algebraic Spaces, Lecture Notes in Mathematics 203 (1971), Springer.

[L-M-B] G. Laumon, L. Moret-Bailly, Champs algébriques, Ergebnisse der Mathematik und ihrer Grenzgebiete. 3. Folge. A Series of Modern Surveys in Mathematics, 39. Springer-Verlag, Berlin, 2000.

[L] Q. Liu, Algebraic Geometry and Arithmetic Curves, Oxford Graduate Texts in Mathematics, Oxford University Press, 2006.

[Ma] B. Margaux, Formal torsors under reductive group schemes, Revista de la Unión Matemética Argentina 60 (2019), 217-224.

[Mn] J. S. Milne, Étale cohomology, Princeton Mathematical Series 33 (1980), Princeton University Press, Princeton, N.J.

[M-B] L. Moret-Bailly, Un problème de descente, Bull. Soc. Math. France 124 (1996), 559-585.

[M-B2] L. Moret-Bailly, Points rationnels dans leur fibre: compléments à un théorème de Poonen, preprint (2019), arXiv:1910.14542

[N] Y.A. Nisnevich, Espaces homogènes principaux rationnellement triviaux et arithmétique des schémas en groupes réductifs sur les anneaux de Dedekind, C.R. Acad. Sc. Paris 299 (1984) 5-8.

[O] M. Olsson, Algebraic spaces and stacks, American Mathematical Society Colloquium Publications, 62. American Mathematical Society, Providence, RI, 2016.

[P] B. Poonen, Points having the same residue field as their image under a morphism, J. Algebra 243 (2001), 224-227. 
[P-S] V. A. Petrov, A.K. Stavrova, Elementary subgroups in isotropic reductive groups, Algebra i Analiz 20 (2008), 160-188; translation in St. Petersburg Math. J. 20 (2009), 625-644.

[Ra] M. Raynaud, Spécialisation du foncteur de Picard, Publications Mathématiques de l'I.H.É.S 38 (1970), 27-76.

[R] C. Riehm, The congruence subgroup problem over local fields, American Journal of Mathematics 92 (1970), 771-778.

[Ry] D. Rydh, Submersions and effective descent of étale morphisms, Bull. Soc. Math. France 138 (2010), 181-230.

[SGA3] Séminaire de Géométrie algébrique de l'I. H. E. S., 1963-1964, schémas en groupes, dirigé par M. Demazure et A. Grothendieck, Lecture Notes in Math. 151-153. Springer (1970).

[SGA4] Séminaire de Géométrie Algébrique du Bois Marie - 1963-64 - Théorie des topos et cohomologie étale des schémas, Lecture Notes in Math. 605. Springer (1972).

[So] C. Sorger, Lectures on moduli of principal G-bundles over algebraic curves, School on Algebraic Geometry (Trieste, 1999), 1-57, ICTP Lect. Notes, 1, Abdus Salam Int. Cent. Theoret. Phys., Trieste, 2000.

[St] Stacks project, https://stacks.math.columbia.edu

[T] J. Tits, Groupes de Whitehead de groupes algébriques simples sur un corps, Séminaire Bourbaki, no. 19 (1976-1977), Talk no. 505, p. 218-236.

UMR 5208 Institut Camille Jordan - Université Claude Bernard Lyon 143 bouleVARD DU 11 NOVEMbre 191869622 Villeurbanne CEDEX - France

E-mail address: gille@math.univ-lyon1.fr

Departement of Mathematics and Computer Science, MSC W401, 400 Dowman Dr. Emory University Atlanta, GA 30322 USA

E-mail address: parimala.raman@emory.edu

Departement of Mathematics and Computer Science, MSC W401, 400 Dowman Dr. EMORY University ATLANTA, GA 30322 USA

E-mail address: suresh@mathcs.emory.edu 